Article

\title{
Energy and Environmental Evaluation of Non-Transparent Constructions of Building Envelope for Wooden Houses
}

\author{
Silvia Vilčeková ${ }^{1, *}$, Monika Čuláková ${ }^{2}$, Eva Krídlová Burdová ${ }^{1}$ and Jana Katunská $^{2}$ \\ 1 Institute of Environmental Engineering, Technical University of Kosice, Vysokoškolská 4, \\ Košice 042 00, Slovakia; E-Mail: eva.kridlova.burdova@tuke.sk \\ 2 Institute of Architectural Engineering, Technical University of Kosice, Vysokoškolská 4, \\ Košice 042 00, Slovakia; E-Mails: monika.culakova@gmail.com (M.Č.); \\ jana.katunska@tuke.sk (J.K.) \\ * Author to whom correspondence should be addressed; E-Mail: silvia.vilcekova@tuke.sk; \\ Tel.: +421-55-602-4260.
}

Academic Editor: Hossam A. Gabbar

Received: 08 July 2015 / Accepted: 28 September 2015 / Published: 2 October 2015

\begin{abstract}
The contribution of embodied energy (EE) and greenhouse gas emissions to building materials and structures has been recognized as significant, especially for nearly-zero energy-efficient buildings. The aim of this paper is to evaluate the composition of non-transparent structures of building envelopes from energy and environmental perspectives using the life-cycle assessment method. The study assesses environmental indicators such as $\mathrm{EE}$ from non-renewable resources and $\mathrm{CO}_{2 \text { eq }}$ and $\mathrm{SO}_{2 \text { eq }}$ emissions from proposed assemblies of building structures for nearly-zero energy wooden houses. Material compositions are also calculated in terms of selected thermal-physical aspects (U-value, phase shift of thermal oscillation, relaxation time) to ensure the reduction of energy consumption during building operation. All results are compared using a multi-dimensional evaluation approach through mathematical methods. The multi-criteria decision analysis demonstrates that material optimization of building structures is possible to ensure a marked reduction of the energy consumption and carbon footprint of buildings.
\end{abstract}

Keywords: wooden houses; building materials; construction; environmental impacts; thermal-physical parameters; multi-criteria decision analysis 


\section{Introduction}

Human beings, like other living creatures, depend on the conditions of the surrounding environment. Therefore, preserving the environment and minimizing negative environmental impacts is a matter of survival [1]. The interaction between society and the environment is a complex web of positive and negative feedback flows. By simplifying the relationship between the natural and social systems, we have, on one side, the flow of natural resources to the system and, on the other side, the flows of waste back to the environment. Damage to the environment depends on its ability to regenerate and capacity for assimilation [2]. Pollutants for which the environment has little or no absorptive capacity create interdependence between the present and the future; the intensity of damage imposed on the future depends on current actions. The present generation is creating a burden for future generations by using up depletable resources and producing pollutants $[2,3]$.

The structure of final energy consumption in 2012 by sector shows that residential, road transport and industry accounted for the biggest shares, $26 \%$ each. The service sector accounted for $13 \%$, transport for $6 \%$ and the other remaining sectors for $3 \%$. Greenhouse gas emissions in the EU-28, stood at 4678.8 million tonnes of $\mathrm{CO}_{2 \text { eq }}$ in 2012. This represents an overall reduction of $17.9 \%$ when compared with 1990, or some 1017 million tonnes of $\mathrm{CO}_{2 \mathrm{eq}}$ [4].

Buildings are one of the leading sectors for energy consumption and emission of greenhouse gas (GHG) production in developed countries. Buildings are responsible for $30 \%-40 \%$ of total primary energy consumption globally [5]. In European Union countries, buildings account for $40 \%-50 \%$ of total energy consumption and are responsible for more than one-third of final GHG emissions throughout their life-cycles. Residential buildings have a key role in the global construction sector; they account for $63 \%$ of total energy consumption and produce $77 \%$ of total $\mathrm{CO}_{2}$ emissions in the European building sector [6-8].

Analysis has revealed that the residential stock is the largest segment of the construction, with $75 \%$ of building stock floor space in the EU. A total of $64 \%$ of residential building floor area is associated with single-family houses and $36 \%$ with apartments. The amount of embodied energy (EE) of a residential building is estimated at $20 \%-40 \%$ of operational energy over its usable life. This differs depending on context due to the type of primary energy resources used, technological advancements, and the methods used for inventory analysis $[7,8]$.

The proper choice of materials for the building envelope plays the important role and can have multiple effects on energy consumption and associated emissions over the different phases of a building's life cycle. EE in building materials can account for $60 \%$ of total life-cycle energy consumption. This can be reduced by applying alternative infill materials, especially insulation in wall systems. The cumulative energy saved over a 50-year life cycle by this material substitution is around $20 \%$ [9]. The identification of the building sector as one of the key consumers of energy led to the creation of rules targeted at improving the energy performance of buildings towards nearly-zero energy performance through the reduction of energy consumption during the occupation phase [10]. This energy consumption of a building is considered the energy used to maintain the occupants' comfort inside the structure (operational energy for energy supply systems). When taking the entire building life-cycle perspective into consideration, the total used energy includes operational and EE [11]. The energy needed for operations can be reduced considerably by improving the insulation of the building 
envelope and through technical solutions. For example phase change materials (PCMs) can be mentioned as suitable construction systems for energy efficient or passive houses. Study [12] dealing with them concluded that PCM passive latent heat thermal energy storage systems can contribute to increase indoor thermal comfort, improve buildings envelope performance and to increase systems efficiency, decrease the conditioning power needed, reduce energy consumption, take advantage of off-peak energy savings, take advantage of renewable sources like solar thermal energy, save money during the operational phase, and contribute for the reduction of $\mathrm{CO}_{2}$ emissions associated to heating and cooling.

Environmental analysis of the life-cycle impacts of a single-family house demonstrated that exterior walls were by far the most significant construction component, with $35 \%$ of $\mathrm{EE}$ and $43 \%$ of $\mathrm{CO}_{2 \text { eq }}$ emissions associated with the construction phase [13]. The embodied environmental impacts such as EE are not considered in current requirements for new buildings. Embodied impacts of building materials and components achieve high values, especially in the case of extremely energy-efficient dwellings. The appropriate selection of building materials during the design process plays a significant role during the life cycle of a building and can affect the sustainability of the project [3]. For dwellings designed according to conventional energy standards, EE is equivalent to a few years of operational energy [14].

On one hand, nearly-zero energy buildings ensure the reduction of operational energy; on the other hand, additional energy is often required for the production of increased amounts of materials and other components, which results in a greater proportion of EE and emissions in the overall lifecycle [15]. High-energy-intensity components are often subject to a wide range of replacements [16]. A comparative study of steel beams at the airport outside Oslo demonstrated that the total energy consumed for manufacturing the beams was two to three times higher, and the use of fossil fuels six to twelve times higher than for manufacturing of glulam beams [17]. The substitution of some building materials showed that EE can be decreased by approximately $17 \%$ [18].

The best chance for reduction of overall intensity of environmental loads is during the integrated design phase of building. The selection of materials has a significant impact on the total energy balance and the GHG emissions of buildings. The EE in building materials and the energy associated with construction and maintenance should be taken into account as they can account for a large share of life-cycle energy consumption [19]. An environmental assessment of three different structural materials for the same house (timber, concrete, and light steel framing) revealed that the timber solution achieved the best scores for all the evaluated environmental aspects. Timber buildings have a greater advantage in the low-energy processes required for the manufacture of materials compared to carbon storage when considering the entire lifecycle [20]. A study of GHG emissions of residential buildings in Sweden concluded that the timber solution decreases carbon emissions two to three times more than the concrete solution, considering that wood waste and logging residues are used to replace fossil fuels [21]. A case study from China of the environmental impacts throughout the building lifecycle demonstrated that energy consumption comprises $70 \%-80 \%$ of the total environmental impact during a 50 -year building lifecycle. Of this amount, the operational phase accounts for $80 \%$, the building material production phase accounts for $15 \%$, and other phases account for the remaining 5\% [22]. Increasing efforts to reduce operational energy consumption in buildings may result in more EE from building materials (mainly insulation materials). Consequently, the relative significance of material 
selection (especially for the envelope of high-energy performance residential buildings) tends to rise $[13,23]$. The analysis of the life cycle of four reference dwellings in Belgium revealed that when energy consumption is reduced, EE increases. However, an increase of EE from 20 to $510 \mathrm{MJ} / \mathrm{m}^{3}$ leads to reduction in energy consumption from 5.5 to $26 \mathrm{GJ} / \mathrm{m}^{3}$ during 30 years of occupation depending on the dwelling and energy performance level. However, only extremely energy-efficient dwellings reached achieved a total EE value higher than the value of energy consumption during operation. Nevertheless, the sum of both EE and primary energy consumption remains much smaller for extremely low-energy dwellings than for average dwellings [24].

In addition to $\mathrm{EE}$, the production of $\mathrm{CO}_{2 \mathrm{eq}}$ emissions plays an important role in the total life cycle of nearly-zero energy buildings. The analysis of environmental impacts of the UK residential sector demonstrated that this sector produces 132 million tons of $\mathrm{CO}_{2 \text { eq }}$ per year; semi-detached houses contributed $40 \%$, terraced houses contributed $37 \%$, and detached houses contributed $27 \%$. Over a 50 -year period, the total GWP from the sector is nearly 6.6 billion tons of $\mathrm{CO}_{2 \mathrm{eq}}$ [25]. However, some case studies of conventional buildings have demonstrated that operational energy is by far the most important contributor to their life-cycle impacts. In contrast, other cases have underlined that, especially for new low-energy buildings, the relative role and importance of life-cycle phases are changing [26]. According to one study [27] a reinforced concrete block masonry structure is a greener option than many traditional building structures, with $\mathrm{CO}_{2}$ reductions of $38-64 \mathrm{~kg} / \mathrm{m}^{2}(6 \%-10 \%)$ and $112 \mathrm{~kg} / \mathrm{m}^{2}$ $(18 \%)$ for concrete structure and brick-concrete structures, respectively, at the materialization stage. In addition, material preparation and daily use were found to be the main carbon sources, contributing $14 \%-17 \%$ and $82 \%-86 \%$, respectively. In another study [28], the $\mathrm{CO}_{2}$ equivalent emissions originating from building materials and products is between 2.4 to 3.1 times higher compared to $\mathrm{CO}_{2}$-equivalent emissions originating from building energy use during operation when the building facade was non-wooden and the service life was 50 years. A different study [29] that employed a hybrid life-cycle assessment (LCA) approach demonstrated that when the temporal allocation of emissions from the construction and use phases is taken into account, construction-phase emissions play a central role in finding effective GHG mitigation strategies - even when the emissions from all consumption activities during the use phase are included in the assessment.

Construction of buildings has a significant impact on energy consumption. Study [30] provides a detailed examination of the energy and greenhouse gas emissions associated with the construction of structural building assemblies. This study states that the construction energy and greenhouse gas emissions vary considerably from one structural system to another and considerably between the major material categories. For both wood and steel structural assemblies, the construction energy is a lower proportion of the total embodied energy than typically assumed and concrete assemblies higher. However, for greenhouse gas emissions, the construction process for concrete assemblies represents a considerably larger proportion of the embodied figure. According to study [31] the energy consumption for construction is 8-10 GJ per $\mathrm{m}^{2}$ of floor area for multi-family steel reinforced concrete houses, 3 GJ for wooden single-family houses, 4.5 GJ for light-weight steel-structure single-family houses. Energy consumption due to construction of a wooden house is approximately $1 / 3$ of that of a steel reinforced concrete multi-family house and $60 \%$ of that for a lightweight steel-structure single-family house. This study also emphasizes that in term of the construction, the wooden house has less impact on the global environment. 
Another study [32] demonstrates the life cycle primary energy balance of the four residential building types (detached house, row house, townhouse and apartment block) based on valid building codes and common building services in Finland. The results of this study are: (i) there are clear differences between the housing types: The detached house is the highest energy consumer, the row house the second (about 20\% less), the townhouse the third (about 30\% less) and the apartment block the lowest (about 45\% less); (ii) the differences appear evenly among the building life cycle stages; (iii) the influence of structural material selection is relatively minor compared to the differences in the housing types; (iv) in principle, the life cycle energy efficiency of a building increases as the number of stories and floor area increase. According to one study [33] the total embodied energy of building materials has been calculated to be equal to $780.1 \mathrm{GJ}$ for the A1 alternative and 698.4 GJ for the A2 alternative (both are masonry houses). The average values of the building materials environmental parameters per floor area were calculated as $4.2 \times 103 \mathrm{MJ} / \mathrm{m}^{2}$ for embodied energy, $202.5 \mathrm{~kg} \mathrm{CO}$ 2eq $/ \mathrm{m}^{2}$ for embodied $\mathrm{CO}_{2}$ emissions, and $1.36 \mathrm{~kg} \mathrm{SO} \mathrm{S}_{2 \mathrm{q}} / \mathrm{m}^{2}$ for embodied $\mathrm{SO}_{2}$ emissions. The average values of the building materials environmental parameters per $1 \mathrm{~kg}$ of used materials were calculated as $2.19 \mathrm{MJ} / \mathrm{kg}$ for embodied energy, $0.105 \mathrm{~kg} \mathrm{CO} 2 \mathrm{eq} / \mathrm{kg}$ for embodied $\mathrm{CO}_{2}$ emissions, and $0.71 \times 10^{-3} \mathrm{~kg}$ $\mathrm{SO}_{2 \mathrm{eq}} / \mathrm{kg}$ for embodied $\mathrm{SO}_{2}$ emissions.

Low energy design of urban environment and buildings in densely populated areas requires consideration of a wide range of factors, including urban setting, transport planning, energy system design, and architectural and engineering details [34].

The aim of the present study is to analyze implementation of sustainable principles in the material-selection, decision-making process. The analysis investigates the role of different building materials in the composition of non-transparent structures of building envelopes in terms of the EE and equivalent emissions of $\mathrm{CO}_{2}$ and $\mathrm{SO}_{2}$. Proposed material compositions are designed to be meet the recommended heat transfer coefficient value of $\mathrm{U}=0.15 \mathrm{~W} / \mathrm{m}^{2} \cdot \mathrm{K}$ for nearly-zero-energy wooden houses. Environmental performance of material solutions is calculated by using the LCA assessment method within "cradle to gate" boundaries. The main contribution of the study is underlining that natural materials have a significant part in the reduction of environmental impacts.

\section{Methods of Research}

The flowchart of the research work (Figure 1) introduces the sustainability assessment approach applied to achieve the research objectives. Step 1 indicates the research object, goal, main criteria, and sub-criteria. The research object is non-transparent structures of the building envelope of nearly-zero-energy wooden houses. The focus is on energy and the environmental impacts of building structures. Step 2 is the proposal of alternatives. There are 60 proposed alternatives for external wall assemblies, 51 alternatives for above-the-ground floor structures, 10 alternatives for on-the-ground floor structures, and 55 alternatives for roof structures for evaluation by MCDA. Step 3 is the prioritization of the criteria/sub-criteria, using pair-wise comparison with Saaty's evaluation scale. Decision-making software based on Analytic Hierarchy Process is used. Step 4 is intent on evaluation and ranking of alternatives of nontransparent structures of building envelope. Finally the best alternatives of building structures were identified and recommended for nearly-zero-energy wooden houses. 


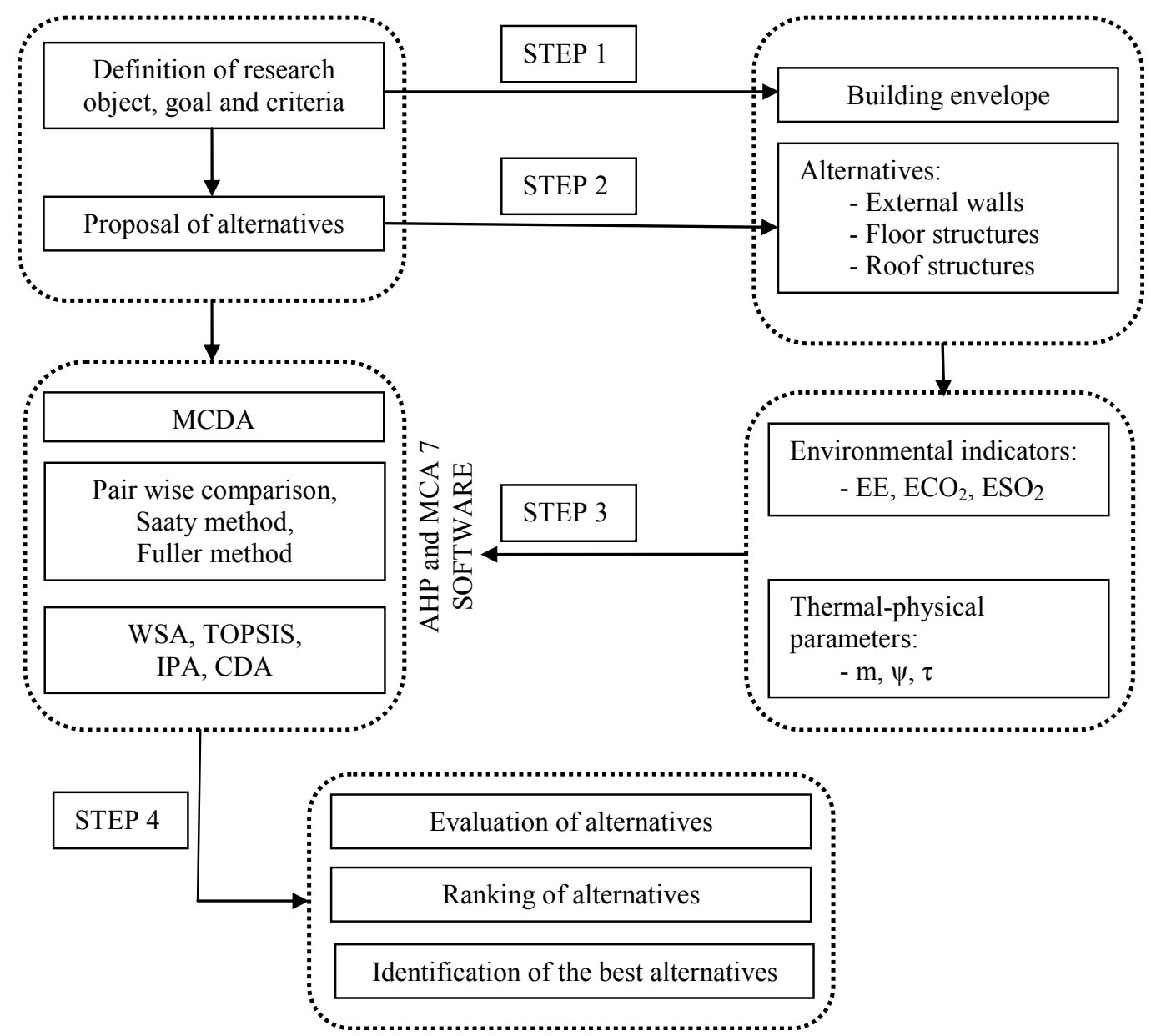

Figure 1. Flowchart of research.

\subsection{Thermo-Physical Parameters}

All material compositions of the non-transparent structure of building envelopes were designed to meet a range of values, including heat transfer coefficient $\mathrm{U}=0.10-0.15 \mathrm{~W} / \mathrm{m}^{2} \cdot \mathrm{K}$ and recommended minimum surface temperature of $16{ }^{\circ} \mathrm{C}$, and to prevent condensation of water vapor inside structures. The subtask was to design material compositions consisting mainly of renewable, natural resources.

The material solutions were designed for nearly-zero-energy houses in Slovakia, which were calculated for climatic conditions according to STN EN 730540:2012 [35]: $\theta_{\mathrm{e}}=-15{ }^{\circ} \mathrm{C} ; \theta_{\mathrm{i}}=20{ }^{\circ} \mathrm{C}$; $\mathrm{R}_{\mathrm{he}}=84 \% ; \mathrm{R}_{\mathrm{hi}}=50 \%$. For the purpose of reduction of future operational energy demand and associated emissions production, the alternative building structure scenarios were compared through selected thermal-physical parameters such as: $U ; \Psi_{e} ; \mathrm{C} ; \psi ; \tau$ and $\theta_{\text {si. }}$.

Most of the aspects were calculated using Svoboda-Area 2009 software according to STN EN 730540:2012. The mathematical calculation of the relaxation time is explained by Equation (1), which depends on the order of material layers. It assigns the ability of the structure to stabilize the inertial temperature during stationary cooling (after turning the heating off) [36]:

$$
\tau=\sum_{i=1}^{n}\left(\frac{d_{i}^{2}}{2 a_{i}}+\frac{\lambda_{i} \cdot d_{i}}{a_{i}} \sum_{j=i+1}^{n} \frac{d_{j}}{\lambda_{j}}\right)
$$

where: $d$-thickness $(\mathrm{m}) ; \lambda$-thermal conductivity coefficient $(\mathrm{W} /(\mathrm{m} \cdot \mathrm{K})) ; a$ - thermal diffusivity $\left(\mathrm{m}^{2} / \mathrm{s}\right)$. 


\subsection{Life-Cycle Assessment}

Environmental performance of material solutions is calculated by using the LCA assessment method within "cradle to gate" boundaries according to the recommendations of EN 15804:2012 [37]. LCA is a standardized tool used to assess and report relevant environmental impacts of a product's life cycle. The LCA framework is interpreted in ISO series 14040-44 [38]. The analysis investigated the role of different building material compositions in terms of EE from non-renewable resources and emissions of $\mathrm{CO}_{2}$ and $\mathrm{SO}_{2}$ in nearly-zero-energy wooden houses. EE is the energy utilized during the manufacturing phase of the building materials and represents the energy used to acquire raw materials (excavation), manufacture, and transport. Similarly, emissions of $\mathrm{CO}_{2}\left(\mathrm{ECO}_{2}\right.$, global warming potential (GWP)) and $\mathrm{SO}_{2}\left(\mathrm{ESO}_{2}\right.$, acidification potential (AP)) represent the equivalent emissions within the LCA "cradle to gate" boundaries and, for plant materials, takes into account $\mathrm{CO}_{2}$ absorbed during the growth stage. The carbon stock is taken into account in the calculation of $\mathrm{CO}_{2}$ emissions. Input data for environmental indicators were extracted from the IBO Ecological Construction Component Catalog of the Austrian LCA database [39]. In this study, the environmental indicator $\Delta \mathrm{OI} 3$ is also calculated. The $\triangle \mathrm{OI} 3$ indicator for a particular building material layer indicates by how many OI3 points that layer of building materials raises the $\mathrm{OI} 3 \mathrm{CON}$ of a structure. In other words, if we eliminate one layer from a structure, the $\mathrm{OI} 3 \mathrm{CON}$ of the structure will decrease by $\Delta \mathrm{OI} 3$ points [40].

\subsection{Multi-Criteria Decision Analysis}

Assessing many (often conflicting) criteria can help to select the optimal alternative for a given building design. The results for particular alternative scenarios are calculated using multi-criteria decision analysis (MCDA). The MCDA method is now widely accepted and popular as a non-monetary assessment method to aid decision-making when dealing with environmental issues in building projects [41]. The MCDA process helps to bridge several fields of expertise simultaneously and brings the possibility of weighting the different criteria in respect to a specific design and building context. Weights of evaluated criteria are calculated by means of Saaty's method by considering the relative level of significance and size of differences in values of criteria for particular alternatives [41,42]. All investigated structures met requirements for highly energy-efficient buildings in terms of U-value and surface temperature and thus these criteria were not taken into account during MCDA. The MCDA total score is calculated using a variety of assessment methods: The method weighted sum approach (WSA), ideal point analysis (IPA), technique for order preference by similarity to ideal solution (TOPSIS), and concordance discordance analysis (CDA). The best resultant WSA and TOPSIS scores are those closest to 1 , and the best IPA score is closest to 0 . The best CDA score is the lowest number. 


\section{Material Compositions}

\subsection{External Walls}

Proposed material compositions are designed to be meet the recommended heat transfer coefficient value of $\mathrm{U}=0.15 \mathrm{~W} / \mathrm{m}^{2} \cdot \mathrm{K}$ for nearly-zero buildings Table 1 describes the bearing system and thermal insulation types for all evaluated external wall assemblies.

Table 1. Description of external wall assemblies.

\begin{tabular}{|c|c|c|}
\hline $\begin{array}{c}\text { Bearing } \\
\text { system }\end{array}$ & \multicolumn{2}{|c|}{ Thermal insulation * (Thickness), Additional thermal insulation } \\
\hline MTS & $\begin{array}{l}\text { 1. hemp }(180 \mathrm{~mm}), \text { hemp with PE }(50 \mathrm{~mm}) \text {, } \\
\text { FB } 210(100 \mathrm{~mm}) \\
\text { 2. flax }(180 \mathrm{~mm}) \text {, flax with PE }(80 \mathrm{~mm}), \\
\text { FB } 270(50 \mathrm{~mm}) \\
\text { 3. fleece }(320 \mathrm{~mm}) \\
\text { 4. FB } 160(160 \mathrm{~mm}), \text { FB } 210(100 \mathrm{~mm}) \\
\text { 5. hemp with PE }(280 \mathrm{~mm}) \text {, rock wool } \\
\\
(50 \mathrm{~mm})\end{array}$ & $\begin{array}{ll}\text { 6. } & \text { flax }(280 \mathrm{~mm}), \text { FB } 50(50 \mathrm{~mm}) \\
7 . & \text { straw } 70(280 \mathrm{~mm}), \text { hemp with PE }(80 \mathrm{~mm}) \\
8 . & \text { cellulosic fiber wood } 180 \mathrm{~mm}), \\
& \text { FB } 50(60 \mathrm{~mm}), \text { FB } 210(60 \mathrm{~mm}) \\
9 . & \text { fleece }(240 \mathrm{~mm}), \text { fleece }(80 \mathrm{~mm}) \\
10 . & \text { cellulosic fiber wood }(140 \mathrm{~mm})+\text { FB } 160 \\
& (140 \mathrm{~mm}), \text { blown cellulose }(60 \mathrm{~mm})\end{array}$ \\
\hline I-profile & $\begin{array}{l}\text { 11. flax }(240 \mathrm{~mm}) \text {, flax with PE }(60 \mathrm{~mm}) \\
\text { 12. I-profile, flax }(240 \mathrm{~mm}), \\
\text { flax with PE }(60 \mathrm{~mm}) \\
\text { 13. straw } 70(400 \mathrm{~mm}), \text { fleece }(50 \mathrm{~mm}) \\
\text { 14. cellulose }(300 \mathrm{~mm}) \text {, fleece }(50 \mathrm{~mm}) \\
15 . \text { straw } 90(400 \mathrm{~mm})\end{array}$ & $\begin{array}{l}\text { 16. cork insulation }(240 \mathrm{~mm}) \text {, FB } 50(60 \mathrm{~mm}) \\
\text { 17. hemp }(240 \mathrm{~mm}) \text {, hemp with PE }(40 \mathrm{~mm}) \text {, } \\
\text { FB } 210(50 \mathrm{~mm}) \\
\text { 18. flax }(300 \mathrm{~mm}) \text {, fleece }(40 \mathrm{~mm}) \\
\text { 19. cellulosic fiber wood }(300 \mathrm{~mm}) \text {, } \\
\text { hemp with PE }(50 \mathrm{~mm}) \\
\text { 20. fleece }(300 \mathrm{~mm})\end{array}$ \\
\hline CLT & $\begin{array}{l}\text { 21. FB } 160(240 \mathrm{~mm}), \text { FB } 50(50 \mathrm{~mm}) \\
\text { 22. cellulosic fiber wood } 240 \mathrm{~mm}) \text {, hemp } \\
\text { with PE }(60 \mathrm{~mm}) \\
\text { 23. hemp }(240 \mathrm{~mm}), \text { FB } 210(40 \mathrm{~mm}) \\
\text { 24. } \\
\text { 25B }(240 \mathrm{~mm}) \text {, fleece }(50 \mathrm{~mm}) \\
\text { FB }(240 \mathrm{~mm}) \text {, fleece }(50 \mathrm{~mm})\end{array}$ & $\begin{array}{l}\text { 26. hemp }(240 \mathrm{~mm}) \text {, hemp with PE }(60 \mathrm{~mm}) \\
\text { 27. cellulosic fiber wood }(200 \mathrm{~mm}), \\
\text { blown cellulose }(60 \mathrm{~mm}), \mathrm{FB} 270(35 \mathrm{~mm}) \\
28 \text { flax }(200 \mathrm{~mm}), \mathrm{FB} 50(80 \mathrm{~mm}) \\
\text { 29. cork insulation }(240 \mathrm{~mm}) \\
\text { 30. straw } 90(360 \mathrm{~mm}), \mathrm{FB} 270(35 \mathrm{~mm})\end{array}$ \\
\hline $\begin{array}{l}\text { wooden } \\
\text { board }\end{array}$ & $\begin{array}{l}\text { 31. blown cellulose }(200 \mathrm{~mm}) \\
\text { 32. FB } 210(160 \mathrm{~mm}) \\
\text { 33. hemp }(240 \mathrm{~mm}) \text {, hemp with PE }(60 \mathrm{~mm}) \\
\text { 34. flax }(140 \mathrm{~mm}) \text {, flax with PE }(50 \mathrm{~mm}) \\
\text { 35. straw } 90(300 \mathrm{~mm}) \\
\text { 36. FB } 160(120 \mathrm{~mm}) \text {, fleece }(60 \mathrm{~mm})\end{array}$ & 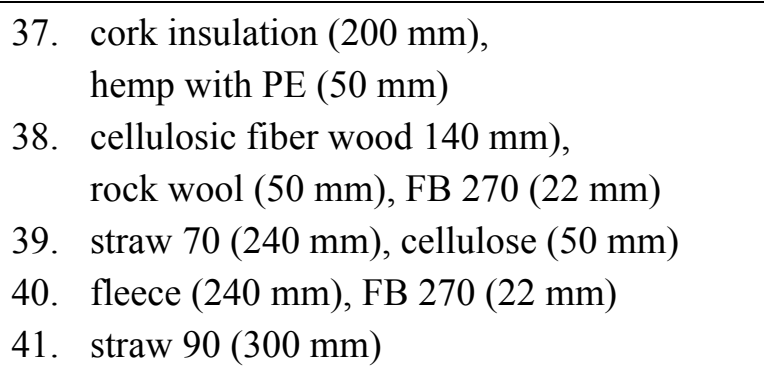 \\
\hline $\begin{array}{c}\text { box } \\
\text { profile }\end{array}$ & $\begin{array}{ll}\text { 42. } & \text { FB } 50(300 \mathrm{~mm}), \text { FB } 50(50 \mathrm{~mm}), \\
& \text { FB } 210(40 \mathrm{~mm}) \\
\text { 43. } & \text { straw } 70(400 \mathrm{~mm}) \\
\text { 44. } & \text { cellulose }(300 \mathrm{~mm}) \text {, rock wool }(50 \mathrm{~mm}) \\
45 . & \text { hemp }(360 \mathrm{~mm}) \\
\text { 46. } & \text { FB } 160(300 \mathrm{~mm}) \text {, fleece }(50 \mathrm{~mm})\end{array}$ & $\begin{array}{l}\text { 47. flax }(300 \mathrm{~mm}) \text {, flax with PE }(60 \mathrm{~mm}) \\
\text { 48. cellulosic fiber wood }(300 \mathrm{~mm}), \\
\text { FB } 210(40 \mathrm{~mm}) \\
\text { 49. blown cellulose }(360 \mathrm{~mm}) \\
\text { 50. hemp }(300 \mathrm{~mm}), \text { FB } 50(50 \mathrm{~mm}), \\
\text { FB } 270(35 \mathrm{~mm})\end{array}$ \\
\hline
\end{tabular}


Table 1. Cont.

\begin{tabular}{|c|c|c|}
\hline $\begin{array}{l}\text { Bearing } \\
\text { system }\end{array}$ & Thermal insulation * (Thickness & , Additional thermal insulation \\
\hline framework & $\begin{array}{l}\text { 51. hemp }(280 \mathrm{~mm}) \text {, hemp with PE }(60 \mathrm{~mm}) \\
\text { 52. cellulose fiber wood }(240 \mathrm{~mm}), \\
\text { FB } 50(60 \mathrm{~mm}) \\
\text { 53. FB } 50(200 \mathrm{~mm}) \text {, fleece }(80 \mathrm{~mm}) \\
\text { FB } 210(40 \mathrm{~mm})\end{array}$ & $\begin{array}{l}\text { 54. straw } 90(350 \mathrm{~mm}) \\
\text { 55. fleece }(300 \mathrm{~mm})\end{array}$ \\
\hline $\log$ house & $\begin{array}{l}\text { 56. FB } 50(240 \mathrm{~mm}), \text { FB } 250(20 \mathrm{~mm}) \\
\text { 57. flax }(220 \mathrm{~mm}), \text { FB } 210(60 \mathrm{~mm}) \\
\text { 58. fleece }(240 \mathrm{~mm}), \text { FB } 270(22 \mathrm{~mm})\end{array}$ & $\begin{array}{l}\text { 59. } \text { straw } 90(360 \mathrm{~mm}) \\
60 . \quad \text { cork insulation }(240 \mathrm{~mm})\end{array}$ \\
\hline
\end{tabular}

* The numbers after the thermal insulation name represents the volume by weight $\left(\mathrm{kg} / \mathrm{m}^{3}\right)$.

\subsection{Floor Constructions}

Due to the application of large amounts of plant materials, above-the-ground floor constructions were designed (51 alternatives) to meet the recommended heat transfer coefficient value of $U=0.10 \mathrm{~W} / \mathrm{m}^{2} \cdot \mathrm{K}$ for nearly-zero buildings. In wooden houses, these constructions are also applied for on-the-ground floors and thus 10 alternative compositions that meet $\mathrm{U}=0.15 \mathrm{~W} / \mathrm{m}^{2} \cdot \mathrm{K}$ were designed. Table 2 briefly describes the material compositions of above-the-ground floors.

Table 2. Description of above-the-ground floor construction assemblies.

\begin{tabular}{|c|c|c|}
\hline Bearing & Thermal insulation * (Thicknes & s), Additional thermal insulation \\
\hline MTS & $\begin{array}{ll}\text { 1. } & \text { cellulosic fiber wood }(320 \mathrm{~mm}), \\
\text { 2. } & \text { fiberboard } 50(400 \mathrm{~mm}) \\
\text { 3. } & \text { hemp with PE }(360 \mathrm{~mm}), \\
& \text { FB } 160(80 \mathrm{~mm}) \\
\text { 4. } & \text { fleece }(380 \mathrm{~mm}), \text { FB } 230(60 \mathrm{~mm}) \\
\text { 5. hemp with PE }(400 \mathrm{~mm}), \\
\text { FB } 160(40 \mathrm{~mm})\end{array}$ & 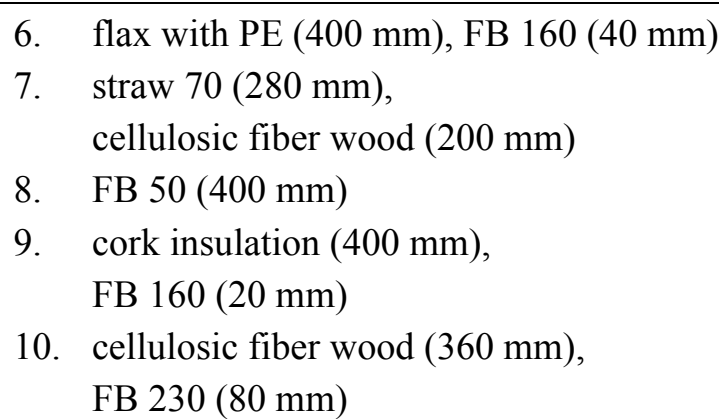 \\
\hline I-profile & $\begin{array}{l}\text { 11. flax with PE }(400 \mathrm{~mm}) \\
\text { 12. hemp with PE }(360 \mathrm{~mm}), \text { hemp }(60 \mathrm{~mm}) \\
\text { 13. FB } 50(360 \mathrm{~mm}), \text { FB } 230(80 \mathrm{~mm}) \\
\text { 14. straw } 90(400 \mathrm{~mm}), \text { FB } 160(50 \mathrm{~mm}) \\
\text { 15. cellulosic fiber wood }(360 \mathrm{~mm}), \\
\text { cellulosic fiber wood }(50 \mathrm{~mm})\end{array}$ & $\begin{array}{l}\text { 16. blown cellulose }(400 \mathrm{~mm}) \\
\text { 17. straw } \rho=70 \mathrm{~kg} / \mathrm{m}^{3}(400 \mathrm{~mm}) \text {, } \\
\mathrm{FB}, 160 \mathrm{~kg} / \mathrm{m}^{3}(60 \mathrm{~mm}) \\
\text { 18. fleece }(400 \mathrm{~mm}) \\
\text { 19. cork insulation }(400 \mathrm{~mm}) \\
\text { 20. hemp with PE }(400 \mathrm{~mm})\end{array}$ \\
\hline $\begin{array}{c}\text { box } \\
\text { profile }\end{array}$ & $\begin{array}{l}\text { 21. hemp with PE }(400 \mathrm{~mm}) \text {, } \\
\text { FB } 160 \mathrm{~kg} / \mathrm{m}^{3}(40 \mathrm{~mm}) \\
\text { 22. fleece }(400 \mathrm{~mm}) \\
\text { 23. blown cellulose }(400 \mathrm{~mm}) \text {, } \\
\text { FB } 160 \mathrm{~kg} / \mathrm{m}^{3}(40 \mathrm{~mm}) \\
\text { 24. hemp with PE }(400 \mathrm{~mm}), \\
\text { FB } 160 \mathrm{~kg} / \mathrm{m}^{3}(20 \mathrm{~mm}) \\
\text { 25. flax with PE }(400 \mathrm{~mm})\end{array}$ & $\begin{aligned} \text { 26. } & \text { cellulosic fiber wood }(360 \mathrm{~mm}), \\
& \text { FB } 160(40 \mathrm{~mm}) \\
27 . & \text { cork insulation }(400 \mathrm{~mm}), \\
& \text { FB } 160(20 \mathrm{~mm}) \\
28 . & \text { straw } 70(400 \mathrm{~mm}), \\
& \text { hemp with PE }(80 \mathrm{~mm}) \\
29 . & \text { FB } 50(400 \mathrm{~mm}) \\
30 . & \text { straw } 90(500 \mathrm{~mm})\end{aligned}$ \\
\hline
\end{tabular}


Table 2. Cont.

\begin{tabular}{|c|c|c|}
\hline CLT & $\begin{array}{l}\text { 31. FB } 50(280 \mathrm{~mm}), \\
\text { cellulosic fiber wood }(100 \mathrm{~mm}) \\
\text { 32. hemp with PE }(280 \mathrm{~mm}), \\
\text { hemp with PE }(120 \mathrm{~mm}) \\
\text { 33. } \\
\text { Flown cellulose }(286 \mathrm{~mm}), \\
\text { 3B160 }(120 \mathrm{~mm}) \\
\text { 35. }\end{array}$ & $\begin{array}{l}\text { 36. cellulosic fiber wood }(360 \mathrm{~mm}) \\
\text { 37. cork insulation }(360 \mathrm{~mm}) \\
\text { 38. blown cellulose }(280 \mathrm{~mm}), \\
\text { cellulosic fiber wood }(100 \mathrm{~mm}) \\
\text { 39. straw } 90(400 \mathrm{~mm}) \\
\text { 40. fleece }(280 \mathrm{~mm}), \text { FB } 50(100 \mathrm{~mm})\end{array}$ \\
\hline $\begin{array}{c}\text { wooden } \\
\text { board }\end{array}$ & $\begin{array}{ll}\text { 41. } & \text { blown cellulose }(280 \mathrm{~mm}) \text {, } \\
& \text { FB } 230(100 \mathrm{~mm}) \\
\text { 42. } & \text { FB } 50(400 \mathrm{~mm}) \\
43 . & \text { hemp }(300 \mathrm{~mm}), \text { hemp with PE }(50 \mathrm{~mm}) \\
44 . & \text { cork insulation }(240 \mathrm{~mm}), \\
\text { FB160 }(50 \mathrm{~mm}) \\
\text { 45. fleece }(360 \mathrm{~mm}) \\
\text { 46. }\end{array}$ & 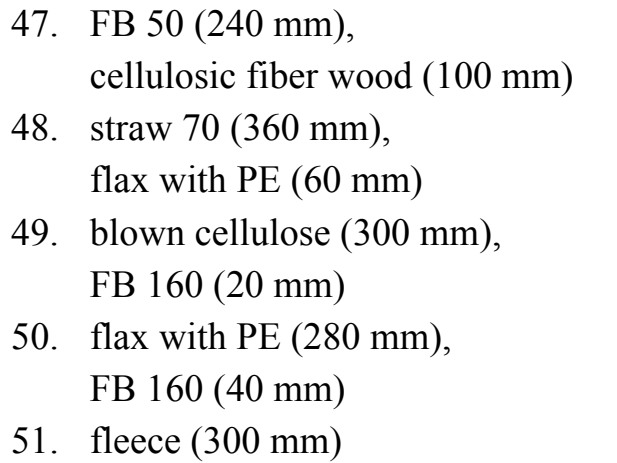 \\
\hline
\end{tabular}

* The number after the thermal insulation name represents the bulk density $\left(\mathrm{kg} / \mathrm{m}^{3}\right)$.

There are 10 proposed compositions of on-the-ground floor structures with a value of $U=0.15 \mathrm{~W} / \mathrm{m}^{2} \cdot \mathrm{K}$. Table 3 describes the evaluated on-the-ground floor structure assemblies.

Table 3. Description of on-the-ground floor construction assemblies.

\begin{tabular}{|c|c|c|}
\hline $\begin{array}{c}\text { Bearing } \\
\text { system }\end{array}$ & \multicolumn{2}{|c|}{ Thermal insulation * (Thickness), Additional thermal insulation } \\
\hline $\begin{array}{l}\text { reinforced } \\
\text { board }\end{array}$ & 52. EPS $30(250 \mathrm{~mm})$ & \\
\hline $\begin{array}{l}\text { concrete } \\
\text { board }\end{array}$ & $\begin{array}{l}\text { 53. XPS-plastic-foam-filled with } \mathrm{CO}_{2}(200 \mathrm{~mm}) \text {, } \\
\text { FB } 230(60 \mathrm{~mm}) \\
\text { 54. EPS with graphite } 35(250 \mathrm{~mm})\end{array}$ & $\begin{array}{l}\text { 55. foam glass compressed }(500 \mathrm{~mm}) \\
56 . \text { foam glass compressed }(400 \mathrm{~mm}) \text {, } \\
\text { FB } 160(50 \mathrm{~mm})\end{array}$ \\
\hline $\begin{array}{l}\text { reinforced } \\
\text { board }\end{array}$ & $\begin{array}{l}\text { 57. foam glass compressed }(250 \mathrm{~mm}) \text {, } \\
\text { FB } 230(100 \mathrm{~mm})\end{array}$ & $\begin{array}{l}\text { 58. foam glass compressed }(350 \mathrm{~mm}) \text {, } \\
\text { FB } 160(80 \mathrm{~mm})\end{array}$ \\
\hline $\begin{array}{c}\text { concrete } \\
\text { board }\end{array}$ & $\begin{array}{l}\text { 59. foam glass compressed }(350 \mathrm{~mm}) \text {, } \\
\text { FB } 160(80 \mathrm{~mm})\end{array}$ & $\begin{array}{l}\text { 60. foam glass compressed }(250 \mathrm{~mm}) \text {, } \\
\text { FB }(100 \mathrm{~mm})+\text { FB } 160(40 \mathrm{~mm})\end{array}$ \\
\hline concrete & 61. XPS-plastic-foam-filled with $\mathrm{CO}_{2}(250 \mathrm{~mm})$ & \\
\hline
\end{tabular}

\subsection{Roof Constructions}

All material compositions of roof constructions were designed to meet the requirements for heat transfer coefficient $\mathrm{U}=0.10 \mathrm{~W} / \mathrm{m}^{2} \cdot \mathrm{K}$ and contain mainly renewable natural materials. Table 4 describes the bearing system and thermal insulation type for all evaluated roof structure assemblies. 
Table 4. Description of roof constructions.

\begin{tabular}{|c|c|c|}
\hline $\begin{array}{c}\text { Bearing } \\
\text { system }\end{array}$ & Thermal insulation * (Thickness) & Additional thermal insulation \\
\hline MTS & $\begin{array}{ll}\text { 1. } & \text { FB } 50(260 \mathrm{~mm}), \text { FB } 50(100 \mathrm{~mm}), \\
& \text { FB } 160(60 \mathrm{~mm})+\text { FB } 270(35 \mathrm{~mm}) \\
\text { 2. } & \text { hemp }(220 \mathrm{~mm})+\text { FB } 50(140 \mathrm{~mm}), \\
\text { hemp with PE }(80 \mathrm{~mm}), \text { FB } 250(60 \mathrm{~mm}) \\
\text { 3. flax }(380 \mathrm{~mm}), \text { FB } 50(50 \mathrm{~mm}) \\
\text { 4. cellulose fiber wood }(380 \mathrm{~mm}), \\
\text { fleece }(60 \mathrm{~mm})\end{array}$ & $\begin{array}{ll}\text { 5. } & \text { cellulose }(260 \mathrm{~mm}) \text {, rock wool }(80 \mathrm{~mm}), \\
& \text { FB } 160(100 \mathrm{~mm}) \\
6 . & \text { fleece }(340 \mathrm{~mm}), \text { hemp with PE } \\
& (100 \mathrm{~mm}), \text { FB } 270(50 \mathrm{~mm}) \\
\text { 7. } & \text { cellulose fiber wood }(280 \mathrm{~mm}), \text { blown } \\
\text { cellulose }(140 \mathrm{~mm}), \text { FB } 270(50 \mathrm{~mm}) \\
8 . \quad \text { straw } 70(280 \mathrm{~mm}), \text { fleece }(100 \mathrm{~mm}), \\
\text { FB } 160(120 \mathrm{~mm})\end{array}$ \\
\hline Glulam & $\begin{array}{l}\text { 9. cellulose fiber wood }(280 \mathrm{~mm}) \text {, } \\
\text { FB } 50(140 \mathrm{~mm}), \text { FB } 270(35 \mathrm{~mm}) \\
\text { 10. hemp }(260 \mathrm{~mm}), \text { hemp }(160 \mathrm{~mm})\end{array}$ & $\begin{array}{l}\text { 11. flax }(200 \mathrm{~mm}) \text {, fleece }(80 \mathrm{~mm}), \\
\text { flax }(140 \mathrm{~mm}) \\
\text { 12. FB } 50(280 \mathrm{~mm}), \text { FB } 50(100 \mathrm{~mm}), \\
\text { FB } 270(50 \mathrm{~mm})\end{array}$ \\
\hline I-profile & $\begin{array}{l}\text { 13. flax }(360 \mathrm{~mm}), \text { FB50 }(60 \mathrm{~mm}) \\
\text { 14. FB } 50(360 \mathrm{~mm}), \text { FB } 50(60 \mathrm{~mm}) \\
\text { FB } 270(35 \mathrm{~mm}) \\
\text { 15. cellulose fiber wood }(360 \mathrm{~mm}) \\
\text { hemp with PE }(80 \mathrm{~mm}) \\
\text { 16. flax }(400 \mathrm{~mm})\end{array}$ & $\begin{array}{l}\text { 18. hemp with PE }(360 \mathrm{~mm}) \text {, } \\
\text { hemp with PE }(60 \mathrm{~mm}) \\
\text { 19. straw } 70(400 \mathrm{~mm}), \text { flax with PE } \\
(80 \mathrm{~mm}), \text { FB } 270(50 \mathrm{~mm}) \\
20 . \quad \begin{array}{l}\text { blown cellulose }(360 \mathrm{~mm}), \\
\text { cellulose fiber wood }(60 \mathrm{~mm})\end{array}\end{array}$ \\
\hline & 17. cork insulation $(400 \mathrm{~mm}), \mathrm{FB} 270(35 \mathrm{~mm})$ & 21. fleece $(400 \mathrm{~mm})$ \\
\hline $\begin{array}{c}\text { box } \\
\text { profile }\end{array}$ & $\begin{array}{l}\text { 22. FB } 160(360 \mathrm{~mm}) \text {, } \\
\text { hemp with PE }(100 \mathrm{~mm}) \\
\text { 23. FB } 50(360 \mathrm{~mm}) \text {, rock wool }(80 \mathrm{~mm}), \\
\text { FB } 270(22 \mathrm{~mm}) \\
\text { 24. flax }(360 \mathrm{~mm}) \text {, flax with PE }(80 \mathrm{~mm}) \\
\text { 25. cellulose fiber wood }(360 \mathrm{~mm}), \\
\text { fleece }(80 \mathrm{~mm}) \\
\text { 26. hemp }(360 \mathrm{~mm}), \text { hemp with PE }(800 \mathrm{~mm}), \\
\text { FB } 270(35 \mathrm{~mm})\end{array}$ & $\begin{array}{l}\text { 27. cork insulation }(400 \mathrm{~mm}) \\
\text { 28. straw } 70(450 \mathrm{~mm}), \text { FB50 }(80 \mathrm{~mm}) \\
\text { 29. fleece }(400 \mathrm{~mm}), \text { FB } 270(35 \mathrm{~mm}) \\
\text { 30. blown cellulose }(360 \mathrm{~mm}), \\
\text { fleece }(80 \mathrm{~mm}) \\
\text { 31. blown cellulose }(450 \mathrm{~mm}) \\
\text { 32. straw } 90(450 \mathrm{~mm}), \text { fleece }(50 \mathrm{~mm}) \\
\text { 33. }\end{array}$ \\
\hline $\begin{array}{l}\text { CLT } \\
\text { panel }\end{array}$ & $\begin{array}{l}\text { 34. flax }(280 \mathrm{~mm}) \text {, flax with PE }(100 \mathrm{~mm}), \\
\text { FB } 270(50 \mathrm{~mm}) \\
\text { 35. cellulose fiber wood }(280 \mathrm{~mm}), \\
\text { fleece }(100 \mathrm{~mm}), \text { FB } 270(50 \mathrm{~mm}) \\
\text { 36. FB } 50(280 \mathrm{~mm}) \text {, FB } 50(100 \mathrm{~mm}), \\
\text { FB } 270(50 \mathrm{~mm}) \\
\text { 37. blown cellulose }(286 \mathrm{~mm}), \text { FB } 50(140 \mathrm{~mm}) \\
\text { 38. fleece }(280 \mathrm{~mm}) \text {, fleece }(100 \mathrm{~mm}), \\
\text { FB } 270(50 \mathrm{~mm})\end{array}$ & $\begin{array}{l}\text { 39. straw } 70(400 \mathrm{~mm}), \\
\text { hemp with PE }(80 \mathrm{~mm}) \\
\text { 40. cork insulation }(400 \mathrm{~mm}) \\
\text { 41. hemp with PE }(280 \mathrm{~mm}), \text { hemp with } \\
\text { PE }(100 \mathrm{~mm}), \text { FB } 270(50 \mathrm{~mm}) \\
\text { 42. blown cellulose }(400 \mathrm{~mm}) \\
\text { 43. hemp with PE }(400 \mathrm{~mm}), \\
\text { FB } 270(50 \mathrm{~mm}) \\
\text { 44. FB } 50(380 \mathrm{~mm}), \text { FB } 50(80 \mathrm{~mm}), \\
\text { FB } 270(50 \mathrm{~mm}) \\
\text { 45. flax with PE }(400 \mathrm{~mm})\end{array}$ \\
\hline $\begin{array}{l}\text { wooden } \\
\text { board }\end{array}$ & $\begin{array}{l}\text { 46. cork insulation }(360 \mathrm{~mm}) \\
\text { 47. blown cellulose }(300 \mathrm{~mm}), \mathrm{FB} 50(60 \mathrm{~mm}) \\
\text { 48. cellulose fiber wood }(240 \mathrm{~mm}), \\
\text { rock wool }(80 \mathrm{~mm}), 250(40 \mathrm{~mm}) \\
\text { 49. straw } 90(400 \mathrm{~mm}) \\
50 . \\
51 . \\
\text { 51. }\end{array}$ & $\begin{array}{l}\text { 52. blown cellulose }(360 \mathrm{~mm}) \\
\text { 53. fleece }(280 \mathrm{~mm}) \text {, fleece }(80 \mathrm{~mm}) \\
54 . \text { flax }(240 \mathrm{~mm}) \text {, flax with PE }(80 \mathrm{~mm}) \text {, } \\
\text { FB } 270(35 \mathrm{~mm}) \\
\text { 55. cellulose fiber wood }(300 \mathrm{~mm}), \\
\text { FB } 210(40 \mathrm{~mm})\end{array}$ \\
\hline
\end{tabular}

* The number after the thermal insulation name represents the volume by weight $\left(\mathrm{kg} / \mathrm{m}^{3}\right)$. 


\section{Results and Discussion}

The described methods were used to determine environmental and thermal-physical parameters for alternative structure designs for building envelopes that are suitable for nearly-zero-energy wooden houses. The alternatives were ranked by calculating the significant weights of evaluated parameters. The results are presented in Table 5.

Table 5. Final relative significance weighting of determined indicators.

\begin{tabular}{cc}
\hline Indicator & Weights (\%) \\
\hline $\mathrm{EE}$ & 17.98 \\
$\mathrm{ECO} 2$ & 17.98 \\
$\mathrm{ESO} 2$ & 8.99 \\
$\mathrm{~m}$ & 3.27 \\
$\tau$ & 25.89 \\
$\psi$ & 25.89 \\
\hline
\end{tabular}

\subsection{External Walls}

Figure 2 illustrates the differences in values of mass per unit area, phase shift of thermal oscillation, and relaxation time for external wall designs.
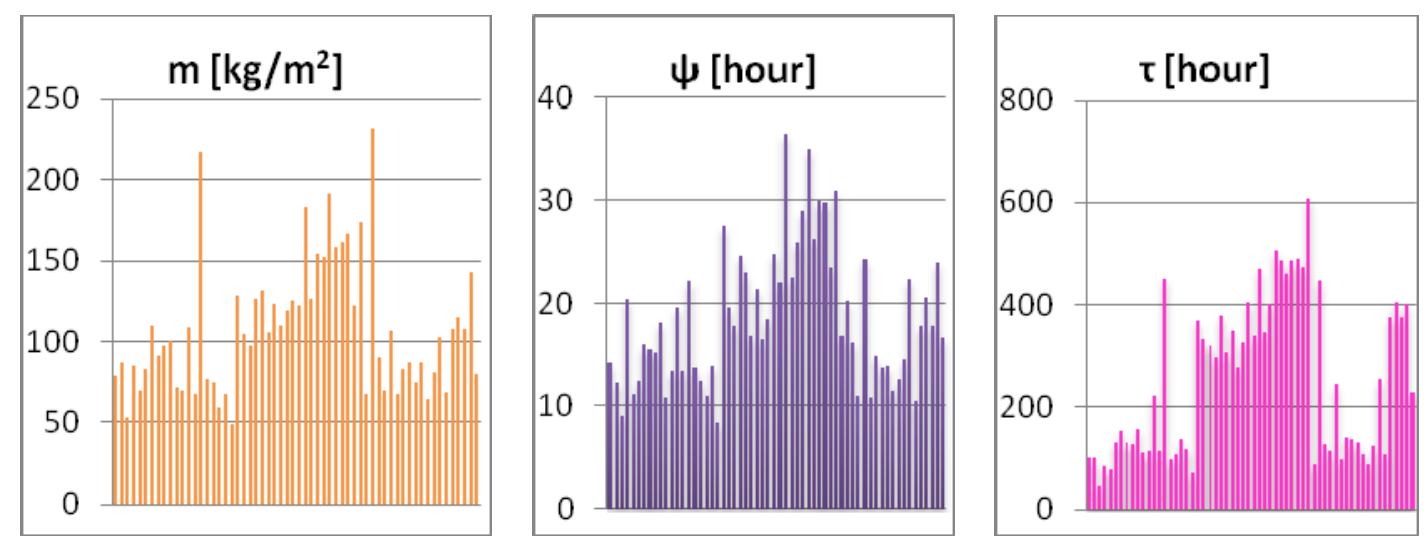

Figure 2. Mass per unit area, phase shift of thermal oscillation, and relaxation time of external walls.

Figure 3 shows that all the designed external wall compositions reached a negative overall balance of $\mathrm{CO}_{2 \text { eq }}$ emissions. The reasons for this are the amount of plant materials and consideration of LCA system boundaries. Alternative 20 reached the lowest EE value. This composition consists of gypsum board, OSB board, fleece between I-beams, and wood paneling. This external wall composition reached the second lowest $\mathrm{SO}_{2 \text { eq }}$ emissions value. In terms of this indicator, Alternative 3, designed from gypsum board, vapor barrier, fleece between MTS profiles, and DHF boards, achieved the best results and as the second lowest energy efficiency value. The disadvantage is that these compositions show lower values of phase shift of thermal oscillation and relaxation time. Alternative 32, designed from massive wood and fiberboard insulation panels $\left(210 \mathrm{~kg} / \mathrm{m}^{3}\right)$, reached maximum surface heat capacity and phase shift thermal oscillations values. Alternative 41, which consisted of clay plaster, 
a massive wood panel, straw bales between I-profiles, DHF board, and wood paneling, had the best results for relaxation time and $\mathrm{CO}_{2 \mathrm{eq}}$ emissions. In terms of environmental indicator results of OI3CON, none of the compositions had minimum values. However, it should be noted that some compositions achieved very low levels of the evaluated indicators and can be considered environmentally optimized compositions (below 15 points). Alternatives 13, 15, 16, 59 and 60 reached zero values for the OI $3 \mathrm{CON}$ indicators. The main insulation materials for these alternatives are straw, fleece, and granulated cork. The results presented in Figure 3 show that Alternative 21, designed from gypsum board, fiber insulation at a volume of $50 \mathrm{~kg} / \mathrm{m}^{3}$, CLT panels, fiber insulation at a volume of $160 \mathrm{~kg} / \mathrm{m}^{3}$, and external plaster was the worst option, especially in terms of $\mathrm{EE}$ and $\mathrm{SO}_{2 \text { eq }}$ emissions. All material compositions were compared using MCDA. According to this assessment, Alternative 41 (Table 6) is the most environmentally suitable. This alternative was also determined as the best overall by an evaluation that took into account the environmental and thermal-physical parameters (Table 7). Table 7 presents the results for best alternatives, which are external wall compositions designed from massive wood panels with straw, fleece, and fiberboard insulation.
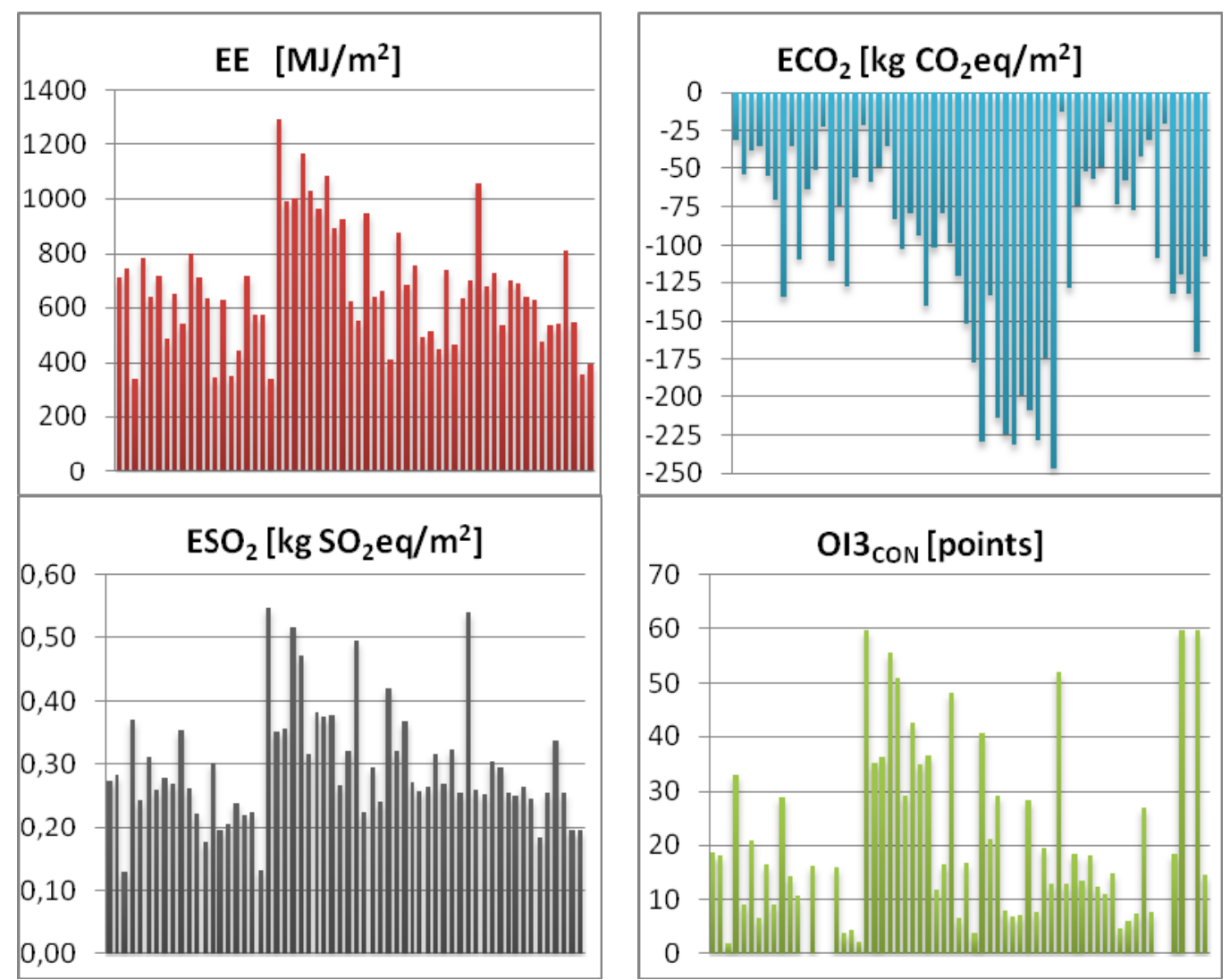

Figure 3. Environmental indicators of material compositions of external walls.

Table 6. Final MCDA scores for environmental evaluation of external walls.

\begin{tabular}{cccccc}
\hline MCDA & $\mathbf{1 .}$ & $\mathbf{2 .}$ & $\mathbf{3 .}$ & $\mathbf{4 .}$ & $\mathbf{5 .}$ \\
\hline WSA & $41(0.888)$ & $35(0.878)$ & $39(0.836)$ & $59(0.829)$ & $15(0.762)$ \\
TOPSIS & $41(0.894)$ & $35(0.886)$ & $39(0.859)$ & $34(0.766)$ & $59(0.748)$ \\
IPA & $41(0.112)$ & $35(0.122)$ & $39(0.164)$ & $59(0.171)$ & $15(0.238)$ \\
CDA & $35(7.481)$ & $41(8.451)$ & $59(9.710)$ & $15(13.677)$ & $39(14.006)$ \\
\hline
\end{tabular}


Table 7. Final MCDA scores for overall evaluation of external walls.

\begin{tabular}{cccccc}
\hline MCDA & $\mathbf{1 .}$ & $\mathbf{2 .}$ & $\mathbf{3 .}$ & $\mathbf{4 .}$ & $\mathbf{5 .}$ \\
\hline WSA & $41(0.879)$ & $35(0.808)$ & $39(0.789)$ & $36(0.728)$ & $32(0,706)$ \\
TOPSIS & $41(0.889)$ & $35(0.815)$ & $39(0.805)$ & $36(0.751)$ & $38(0.744)$ \\
IPA & $41(0.121)$ & $35(0.192)$ & $39(0.211)$ & $36(0.272)$ & $32(0.294)$ \\
CDA & $35(9.104)$ & $41(9.463)$ & $39(12.161)$ & $59(15.072)$ & $40(16.675)$ \\
\hline
\end{tabular}

Figures 4 and 5 illustrate the percentage share and the impact of materials on environmental indicators for the best alternative number 41 .

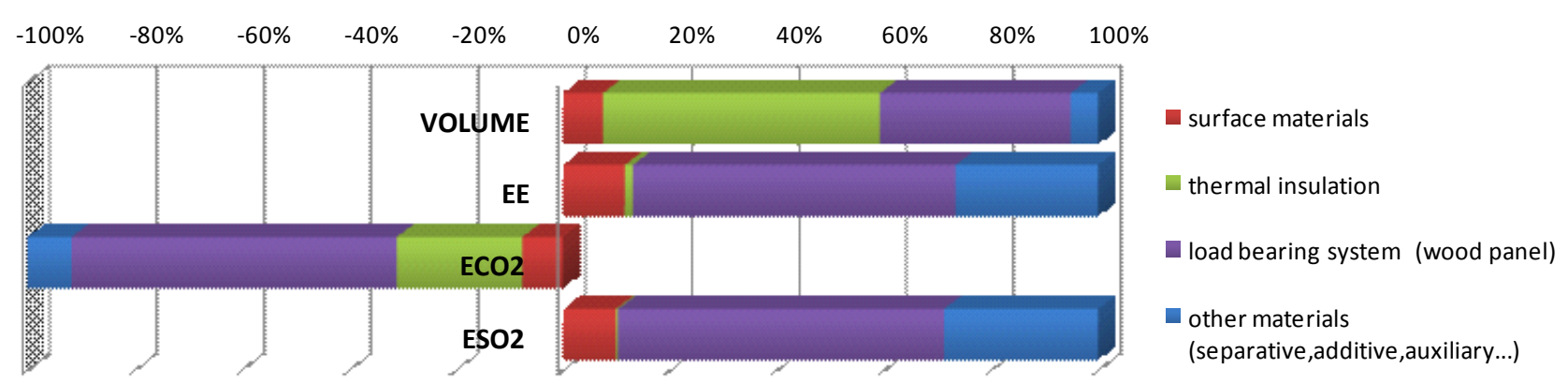

Figure 4. External walls: Materials share by percentage and impact on environmental indicators.
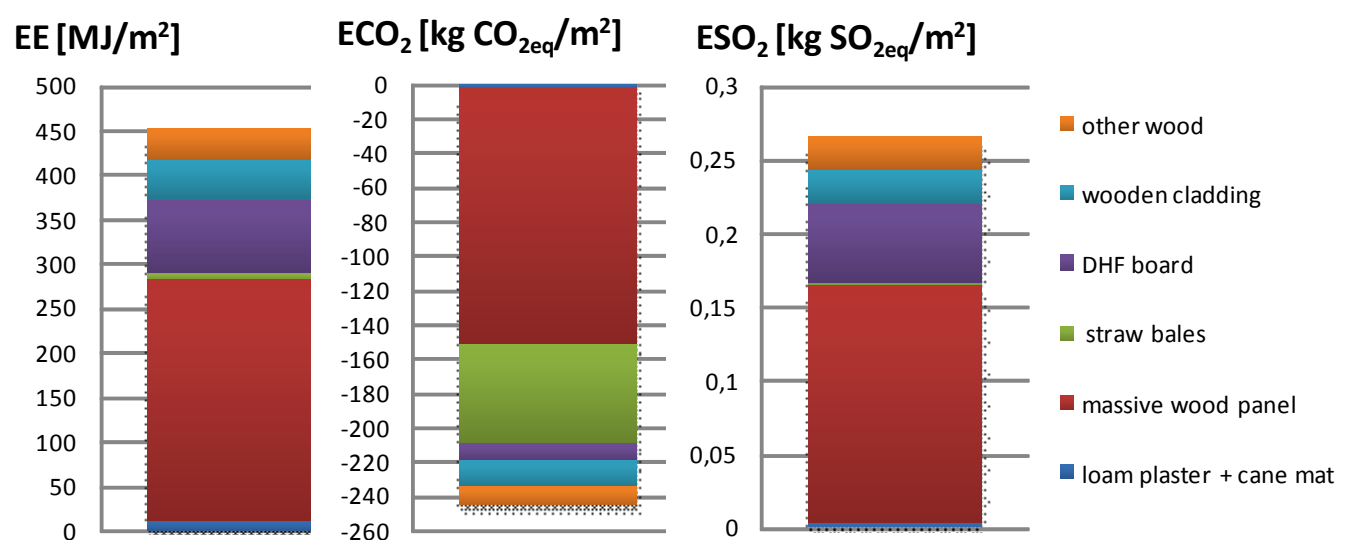

Figure 5. External wall: Impact of materials on environmental indicators.

\subsection{Floor Constructions}

Figure 6 illustrates the differences in values of mass per unit area, phase shift of thermal oscillation, and relaxation time determined for above-the-ground floor designs. 

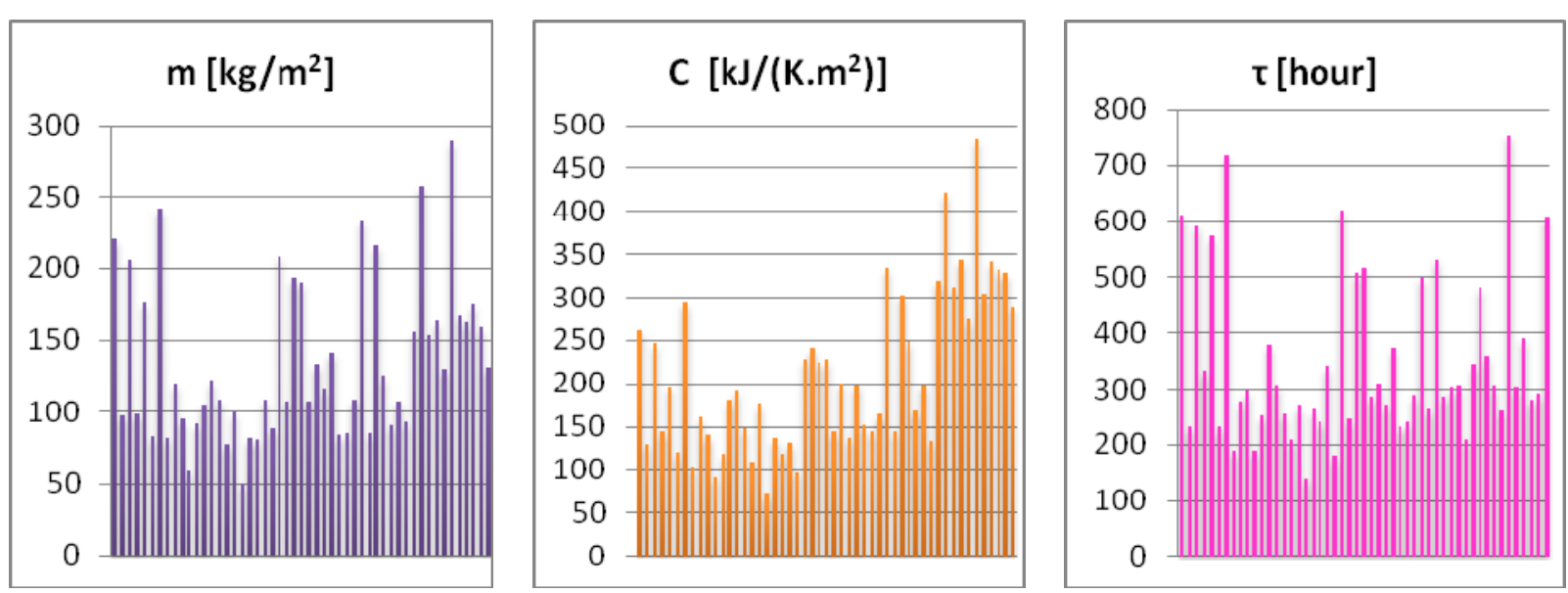

Figure 6. Mass per unit area, phase shift of thermal oscillation, and relaxation time of above-the-ground floor structures.

Figure 7 reveals that all alternatives achieved a negative overall balance of $\mathrm{CO}_{2 \mathrm{eq}}$ emissions. The reasons for this are the amount of natural plant materials and consideration of LCA "cradle to gate" system boundaries, which consider absorption of emissions as plants are growing. Excessive elimination of $\mathrm{CO}_{2 \text { eq }}$ emissions was found in compositions containing of straw insulation, massive wooden panels, and cork insulation. Alternative 18, designed from cork board, OSB boards, fleece thermal insulation between I-profiles, and diffusion fiberboard (DHF), was the best in terms of EE, $\mathrm{SO}_{2 \text { eq }}$ emissions, and $\mathrm{OI} 3 \mathrm{CON}$ environmental indicators (Figure 7) and mass per unit area (Figure 6), but this alternative was one of the worst in terms of total surface heat capacity and relaxation time values. For these two criteria, Alternative 46 was found to be the most effective and achieved the best results for ability to eliminate $\mathrm{CO}_{2 \text { eq }}$ emissions. This alternative consists of cork board, cob brick, OSB boards, thermal insulation of straw bales inserted between I-beams, and a massive wood panel (wood cross-jointed by oak pins). For above-the-ground floor compositions, Alternative 26, which consisted of laminate flooring, insulating belt, self-leveling screed, screed floor cover, PE foil, fiberboard of $160 \mathrm{~kg} / \mathrm{m}^{3}$, OSB boards, cellulose-fiberboard insulation, diffusion foil, and DHF boards, had the most unfavorable EE and environmental indicators values. The effect of increasing stored energy was found mainly in concrete and laminate flooring. Alternative 36, composed of laminate flooring, self-leveling screed floor cover, PE foil, OSB boards, cellulose-fiberboard insulation between KVH profiles, and CLT panels, had a high of stored energy value. The MCDA method was used for the final decision about the best floor structure alternative. Results for the five best alternatives are presented in Tables 8 and 9 .

On the basis of the results presented in Tables 8 and 9, it can be stated that the most appropriate above-the-ground floor structure material assembly is Alternative 46 for both assessments (i.e., environmental evaluation and evaluation from environmental and thermal-physical parameters). Ultimately, based on the total MCDA score, massive wood panels, cob brick, and thermal insulation from straw were determined to be very effective materials. Straw is a renewable agricultural waste material that consumes minimum energy during its treatment. 

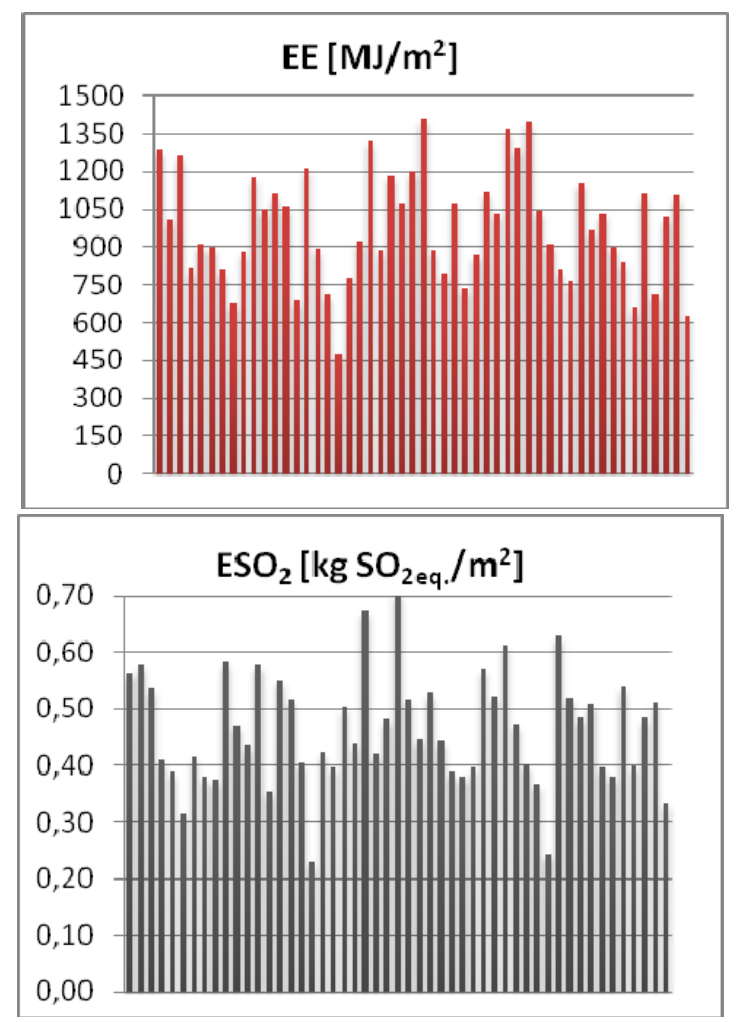

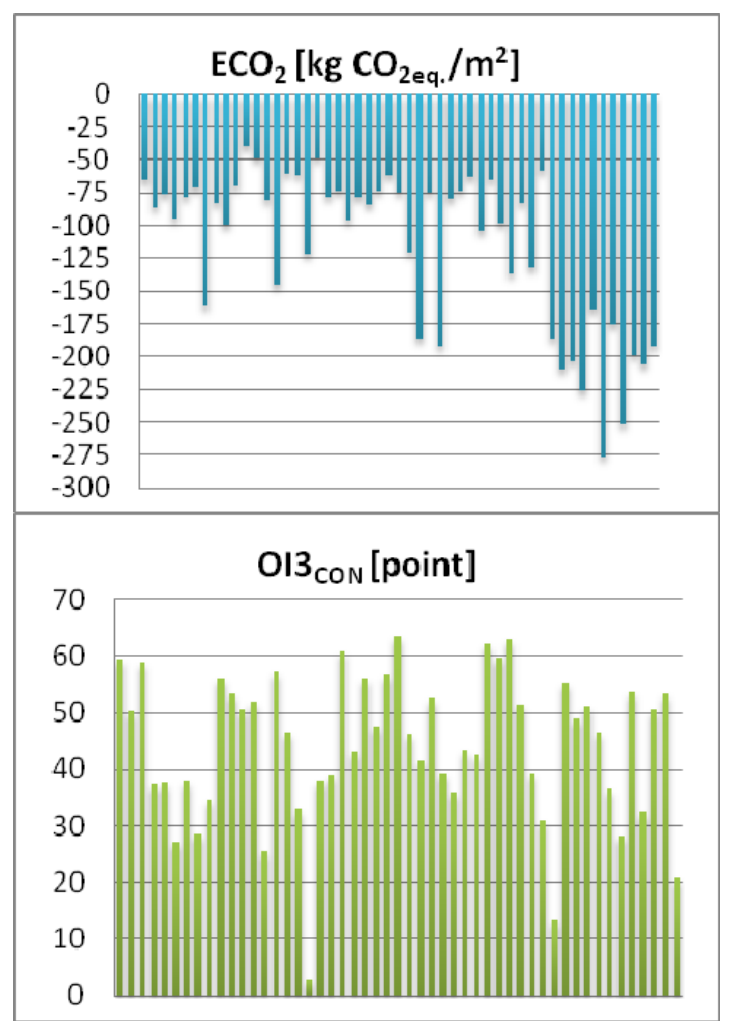

Figure 7. Environmental indicators of material compositions of above-the-ground floor constructions.

Table 8. Final MCDA scores for environmental evaluation of above-the-ground floor structures.

\begin{tabular}{cccccc}
\hline MCDA & $\mathbf{1 .}$ & $\mathbf{2 .}$ & $\mathbf{3 .}$ & $\mathbf{4 .}$ & $\mathbf{5 .}$ \\
\hline WSA & $46(0.895)$ & $48(0.850)$ & $51(0.834)$ & $18(0.765)$ & $14(0.765)$ \\
TOPSIS & $46(0.874)$ & $48(0.844)$ & $51(0.819)$ & $30(0.760)$ & $28(0.765)$ \\
IPA & $46(0.105)$ & $48 .(0.150)$ & $51(0.166)$ & $18(0.235)$ & $14(0.235)$ \\
CDA & $46 .(2.908)$ & $51(5.017)$ & $48(6.809)$ & $14(10.560)$ & $30(12.855)$ \\
\hline
\end{tabular}

Table 9. Final MCDA scores for overall evaluation of above-the-ground floor structures.

\begin{tabular}{cccccc}
\hline MCDA & $\mathbf{1 .}$ & $\mathbf{2 .}$ & $\mathbf{3 .}$ & $\mathbf{4 .}$ & $\mathbf{5 .}$ \\
\hline WSA & $46(0.857)$ & $48(0.785)$ & $51(0.747)$ & $30(0.655)$ & $14(0.636)$ \\
TOPSIS & $46(0.861)$ & $48(0.808)$ & $51(0.711)$ & $30 .(0.661)$ & $44(0.661)$ \\
IPA & $46(0.143)$ & $48(0.215)$ & $51(0.253)$ & $30(0.345)$ & $14(0.364)$ \\
CDA & $46(2.707)$ & $51(5.452)$ & $48(6.704)$ & $14(11.754)$ & $30(12.968)$ \\
\hline
\end{tabular}

Figure 8 illustrates the differences in values of mass per unit area and relaxation time determined for on-the-ground floor. 

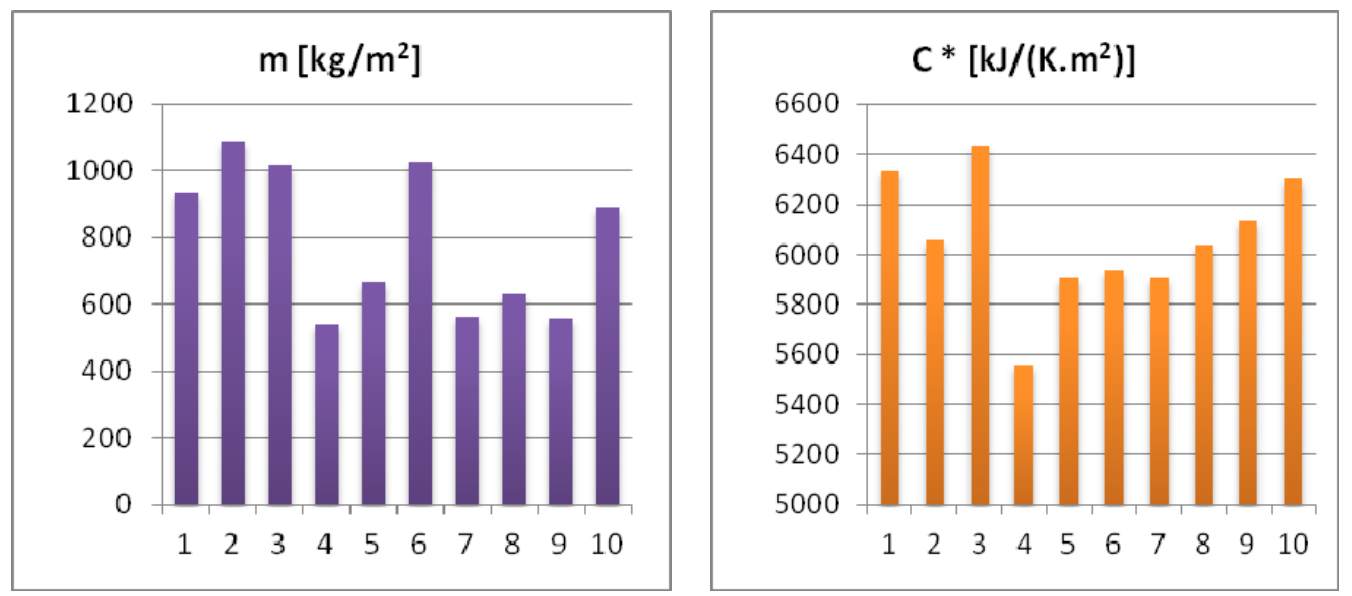

Figure 8. Mass per unit area and phase shift of thermal oscillation of on-the-ground floor structures.

Figure 9 shows that on-the-ground floor structures achieve poorer environmental performance compared to above-the-ground floor structures. Environmental performance could be improved, for example, by applying of large quantities of recycled materials, using construction waste or geopolymer concrete, which is made of recycled scrap, to reinforce the steel, or replacing it with special types of plant fibers. However, input data of environmental indicators for such solutions do not yet exist. Alternative 55, composed of wood flooring, cork insulation roll, concrete slab with a wire net, waterproofing and geotextiles, compressed foam glass (recycled, 98\% waste glass), and separation geotextiles, achieved the best results in terms of $\mathrm{EE}, \mathrm{SO}_{2 \text { eq }}$ emissions, and $\mathrm{OI} 3 \mathrm{CoN}$ environmental indicators. Alternative 60, a floor structure composed of wood floor, fiber insulation with volume density of $160 \mathrm{~kg} / \mathrm{m}^{3}$ between slats, OSB board, fiber insulation of $230 \mathrm{~kg} / \mathrm{m}^{3}$, insurance waterproofing, concrete slab with wire net, waterproofing and geotextiles, compressed foam glass, and separation geotextiles, achieved the smallest $\mathrm{CO}_{2 \text { eq }}$ emissions value, particularly due to the application of fiber insulation. Compared to above-the-ground floors, on-the-ground floors have benefits associated with the positive impact of soil, which is reflected in lower requirements for U-values and higher skills accumulation, which has an impact the concrete slab respectively reinforced concrete slab.
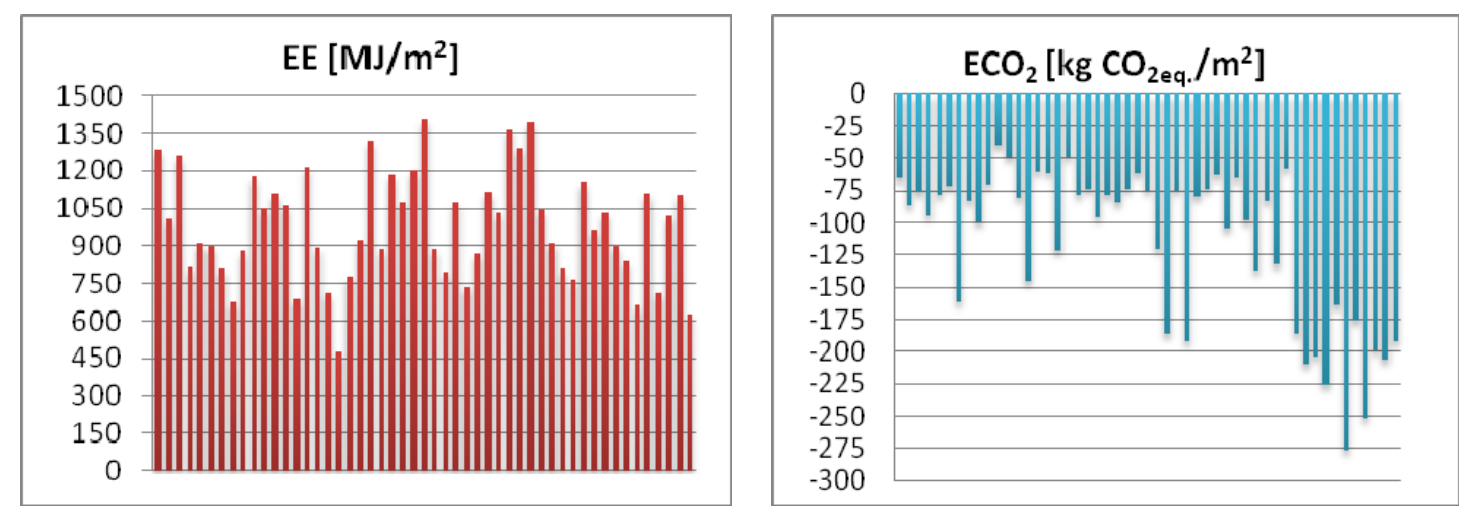

Figure 9. Cont. 

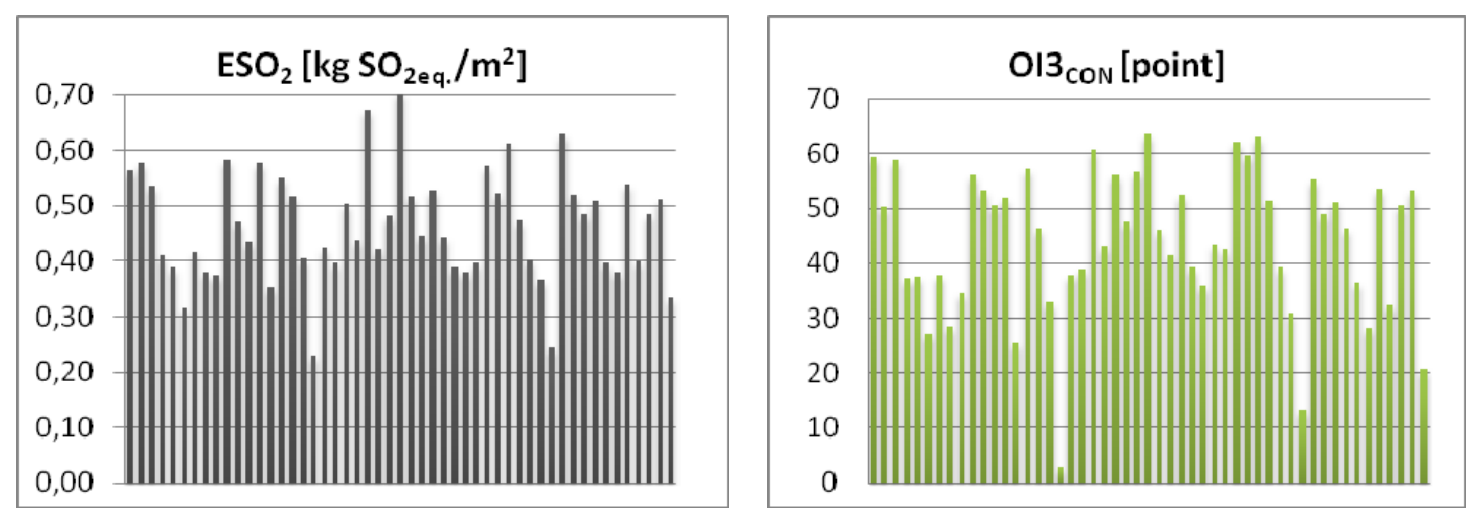

Figure 9. Environmental indicators of material compositions of on-the-ground floor structures.

Based on MCDA, we established the two best on-the-ground floor designs in terms of environmental evaluation and overall evaluation (i.e., evaluation from environmental and thermal-physical parameters) (Table 10). Based on the environmental aspects, the best solution is Alternative 55 (for three methods) and 60 (according to TOPSIS). In both assemblies, foam glass insulation is used, and in Alternative 60, fiber insulation is also used. From a comprehensive analysis, Alternative 60 was proven the best solution for use in wooden houses.

Table 10. Final MCDA scores for on-the-ground floors.

\begin{tabular}{cccccc}
\hline \multicolumn{3}{c}{ Environmental evaluation } & \multicolumn{2}{c}{ Overall evaluation } \\
\hline MCDA & $\mathbf{1 .}$ & $\mathbf{2 .}$ & MCDA & $\mathbf{1 .}$ & $\mathbf{2 .}$ \\
\hline WSA & $55(0.729)$ & $60(0.721)$ & WSA & $60(0.707)$ & $52(0.522)$ \\
TOPSIS & $60(0.728)$ & $55(0.604)$ & TOPSIS & $60(0.728)$ & $55(0.603)$ \\
IPA & $55(0.271)$ & $60(0.279)$ & IPA & $60(0.293)$ & $52(0.478)$ \\
CDA & $55(1.856)$ & $60(2.077)$ & CDA & $60(3.036)$ & $61(4.816)$ \\
\hline
\end{tabular}

All proposed of floor structure alternatives (on-the-ground and above-the-ground) were evaluated using MCDA. In terms of environmental aspects (Table 11), Alternative 46 can be clearly confirmed as the most suitable as well as the most suitable for above-the-ground floors only. From the comprehensive analysis (Table 12), there were ambiguous results for all MCDA methods because of the large differences in the surface heat capacity values for on-the-ground and above-the-ground floors.

Table 11. Final MCDA scores for environmental evaluation of all floor structure alternatives.

\begin{tabular}{cccccc}
\hline MCDA & $\mathbf{1 .}$ & $\mathbf{2 .}$ & $\mathbf{3 .}$ & $\mathbf{4 .}$ & $\mathbf{5 .}$ \\
\hline WSA & $46(0.895)$ & $48(0.850)$ & $51(0.834)$ & $18(0.765)$ & $14(0.765)$ \\
TOPSIS & $46(0.874)$ & $48(0.844)$ & $51(0.819)$ & $30(0.760)$ & $28(0.765)$ \\
IPA & $46(0.105)$ & $48(0.150)$ & $51(0.166)$ & $18(0.235)$ & $14(0.235)$ \\
CDA & $46(2.908)$ & $51(5.017)$ & $48(6.809)$ & $14(10.560)$ & $30(12.855)$ \\
\hline
\end{tabular}


Table 12. Final MCDA scores for overall evaluation of all floor structure alternatives.

\begin{tabular}{cccccc}
\hline MCDA & $\mathbf{1 .}$ & $\mathbf{2 .}$ & $\mathbf{3 .}$ & $\mathbf{4 .}$ & $\mathbf{5 .}$ \\
\hline WSA & $55(0.584)$ & $46(0.556)$ & $60(0.555)$ & $48(0.527)$ & $58(0.516)$ \\
TOPSIS & $60(0.704)$ & $58(0.680)$ & $55(0.680)$ & $52(0.674)$ & $61(0.670)$ \\
IPA & $55(0.416)$ & $46(0.444)$ & $60(0.445)$ & $48(0.473)$ & $58(0.480)$ \\
CDA & $46(17.174)$ & $48(19.631)$ & $51(22.071)$ & $44(26.109)$ & $42(28.335)$ \\
\hline
\end{tabular}

As seen in Table 12, the results according to the TOPSIS method vary the most. In this method only, the Euclidean distance as applied for calculating the distance from the basal to ideal alternatives, which impacted the intensification of the impact from high levels of surface heat capacity for on-the-ground floors. The results of this method were not taken into account, and, according to other methods, it can be stated that highly effective solutions are Alternative 55 (on-the-ground floor) and Alternative 46 (above-the-ground floor). Figures 10 and 11 illustrate the shares by percentage and the impact of materials on environmental indicators for Alternative 46.

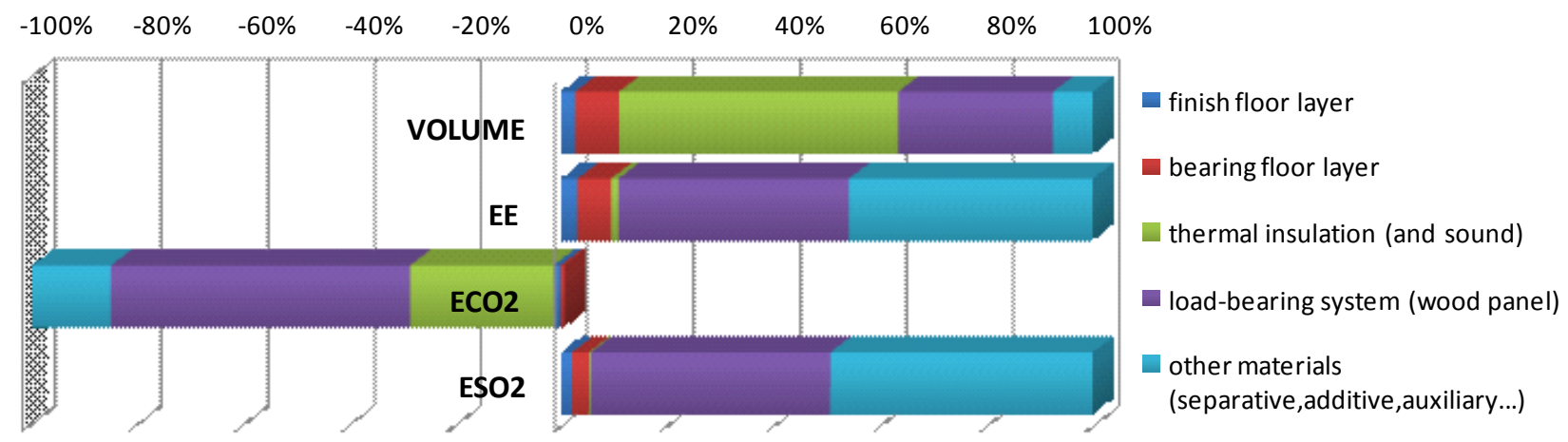

Figure 10. Floor structures: Materials share by percentage and impact on environmental indicators.

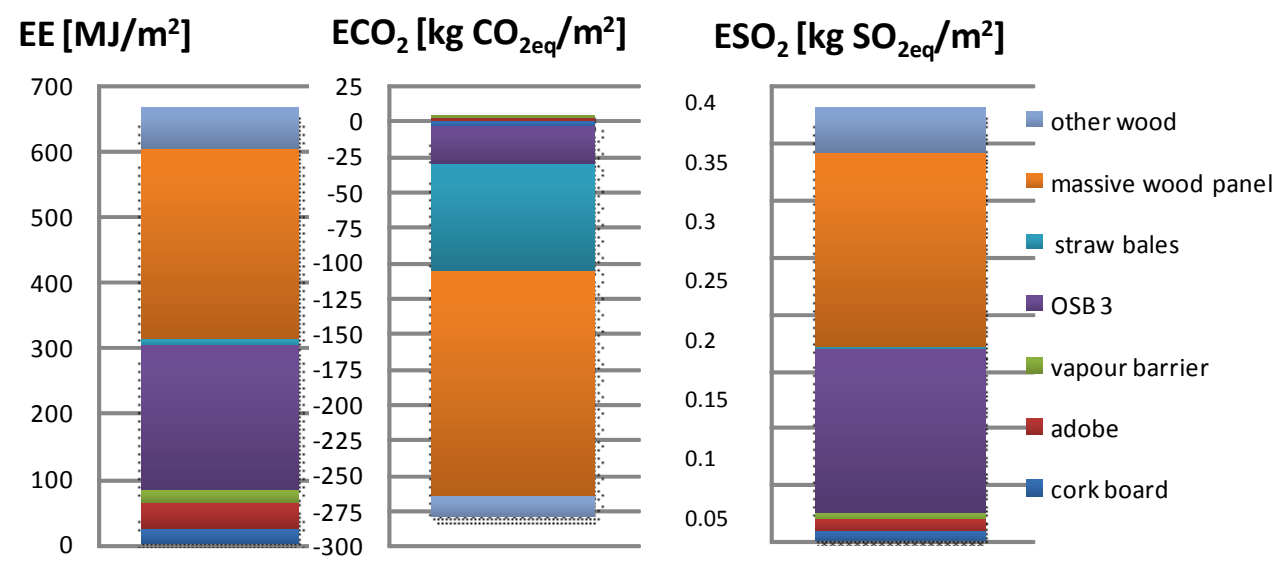

Figure 11. Floor structures: Impact of materials on environmental indicators.

\subsection{Roof Constructions}

Figure 12 illustrates the differences in the mass per unit area, phase shift of thermal oscillation, and relaxation time values determined for the designed roof structures. 

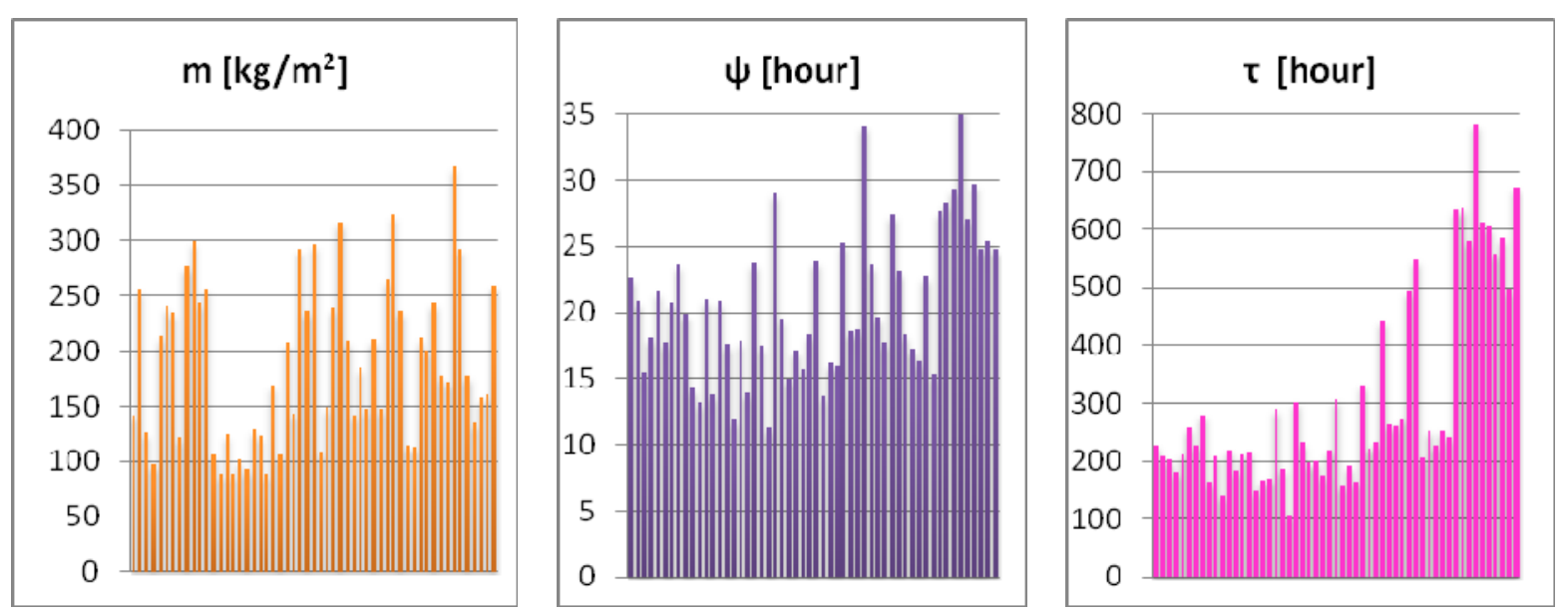

Figure 12. Mass per unit area, phase shift of thermal oscillation, and relaxation time of roof structures.

Based on Figure 13, it can be stated that all alternatives achieved a negative balance of $\mathrm{CO}_{2 \mathrm{eq}}$ emissions, particularly alternatives from massive wood timber. In addition, in this case, the compositions with massive wood panels were determined to be effective solutions from their effects on thermal stability. Alternative 21, which consisted of gypsum board, vapor barrier, wool between I-profiles, DHF board, and roofing in the form of wooden shingles, achieved minimum primary energy and $\mathrm{SO}_{2 \text { eq }}$ emissions values. Alternative 35, which consisted of plasterboard, wool, CLT panels, cellulose-fiberboard insulation between KVH profiles and fiber insulation board, achieved the highest values of both of the above indicators because of the use of titanium-zinc sheet. Alternative 49, which has an extensive green roof and consisted of clay plaster, a massive wood panel, straw bales between I-profiles, DHF boards, wooden formwork, waterproofing, a drainage layer, and substrate, presented the minimum $\mathrm{CO}_{2 \mathrm{eq}}$

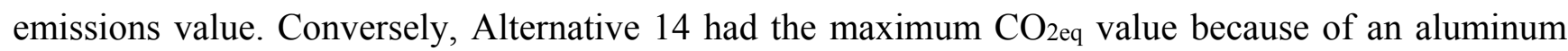
vapor barrier and aluminum sheet covering. After evaluating all alternative roof structure compositions by MCA7, the five best material compositions were determined. These are presented in Tables 13 and 14. Alternative 49 was determined to be the best material composition for both assessments (i.e., environmental evaluation and evaluation based on environmental and thermal-physical parameters). Figures 14 and 15 illustrate the shares by percentage and the impact of materials on environmental indicators for Alternative 49.
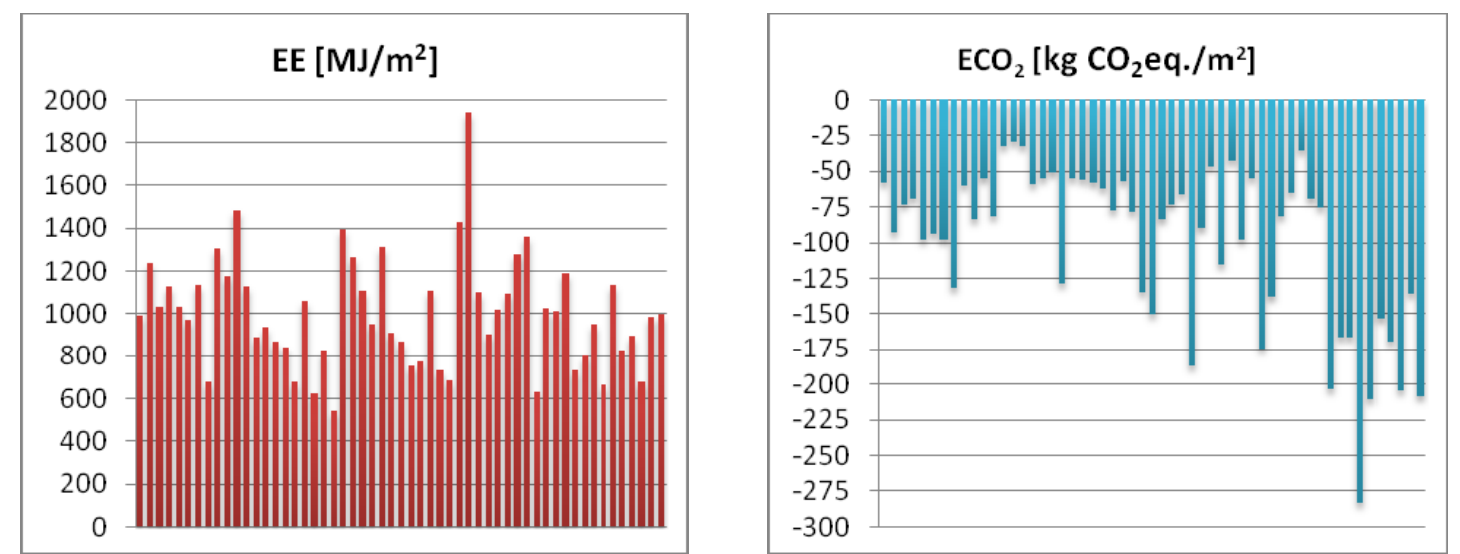

Figure 13. Cont. 

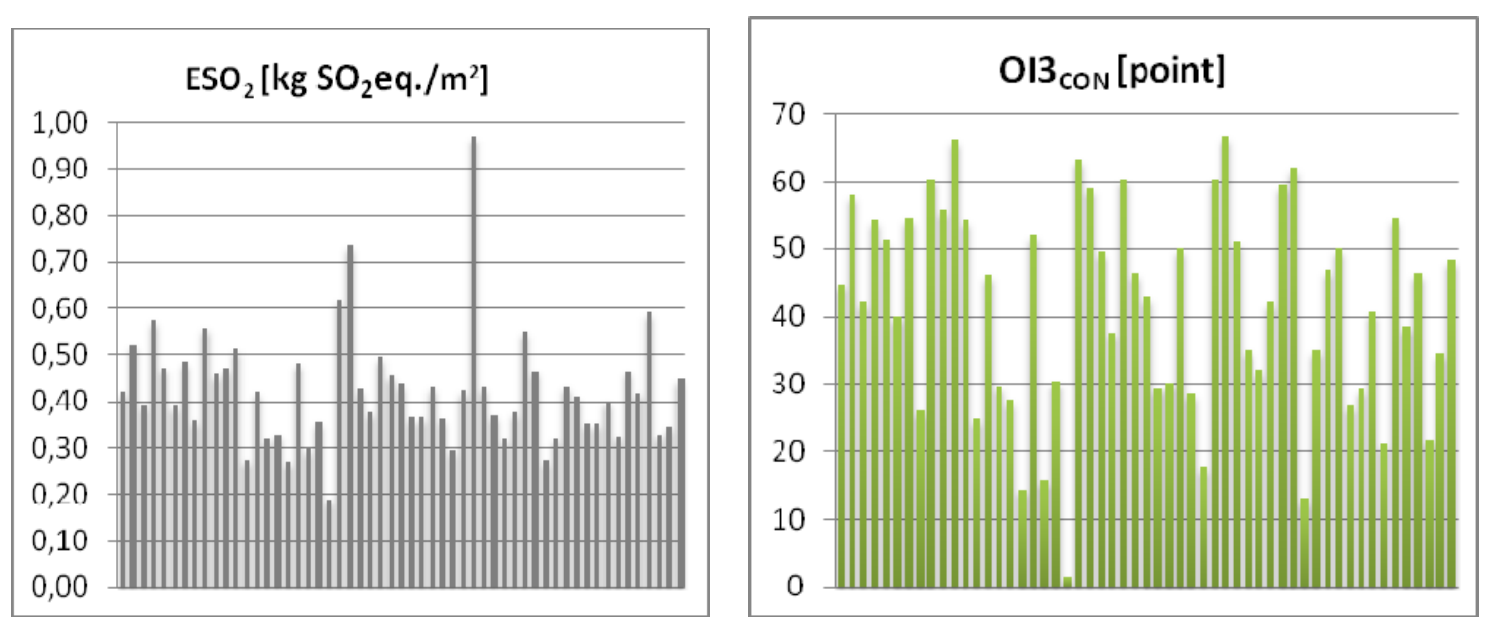

Figure 13. Environmental indicators of material compositions of roof structures.

The main target of this study [43] was to benefit designers and building engineers in their pursuit of finding optimal and competent solutions suitable for specific local microclimates using traditional methods in the design process that are sustainable and ecological. According to this study, the choice of building materials takes a high priority in the building process. Clay, straw, reeds, and brick were the most frequently applied building materials.

Table 13. Final MCDA scores for environmental evaluation of roof structures.

\begin{tabular}{cccccc}
\hline MCDA & $\mathbf{1 .}$ & $\mathbf{2 .}$ & $\mathbf{3 .}$ & $\mathbf{4 .}$ & $\mathbf{5 .}$ \\
\hline WSA & $49(0.931)$ & $53(0.803)$ & $46(0.779)$ & $32(0.750)$ & $19(0.707)$ \\
TOPSIS & $49(0.948)$ & $53(0.730)$ & $46(0.721)$ & $55(0.699)$ & $50(0.679)$ \\
IPA & $49(0.070)$ & $53(0.197)$ & $46(0.221)$ & $32(0.250)$ & $19(0.293)$ \\
CDA & $49(3.252)$ & $53(5.427)$ & $46(8.344)$ & $32(10.310)$ & $19(10.898)$ \\
\hline
\end{tabular}

Table 14. Final MCDA scores for overall evaluation of roof structures.

\begin{tabular}{cccccc}
\hline MCDA & $\mathbf{1 .}$ & $\mathbf{2 .}$ & $\mathbf{3 .}$ & $\mathbf{4 .}$ & $\mathbf{5 .}$ \\
\hline WSA & $49(0.936)$ & $55(0.750)$ & $46(0.743)$ & $47(0.723)$ & $53(0.719)$ \\
TOPSIS & $49(0.935)$ & $55(0.757)$ & $46(0.735)$ & $53(0.702)$ & $50(0.698)$ \\
IPA & $49(0.064)$ & $55(0.250)$ & $46(0.257)$ & $47(0.277)$ & $53(0.281)$ \\
CDA & $49(7.563)$ & $53(9.418)$ & $46(9.748)$ & $47(9.998)$ & $51(12.082)$ \\
\hline
\end{tabular}

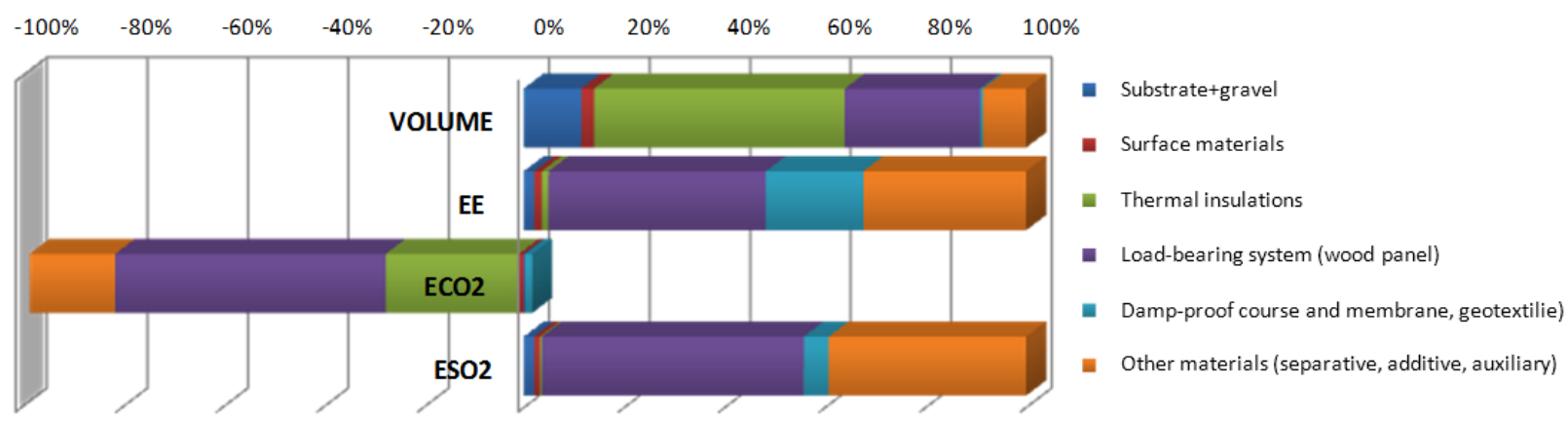

Figure 14. Roof structure: Materials share by percentage and impact on environmental indicators. 


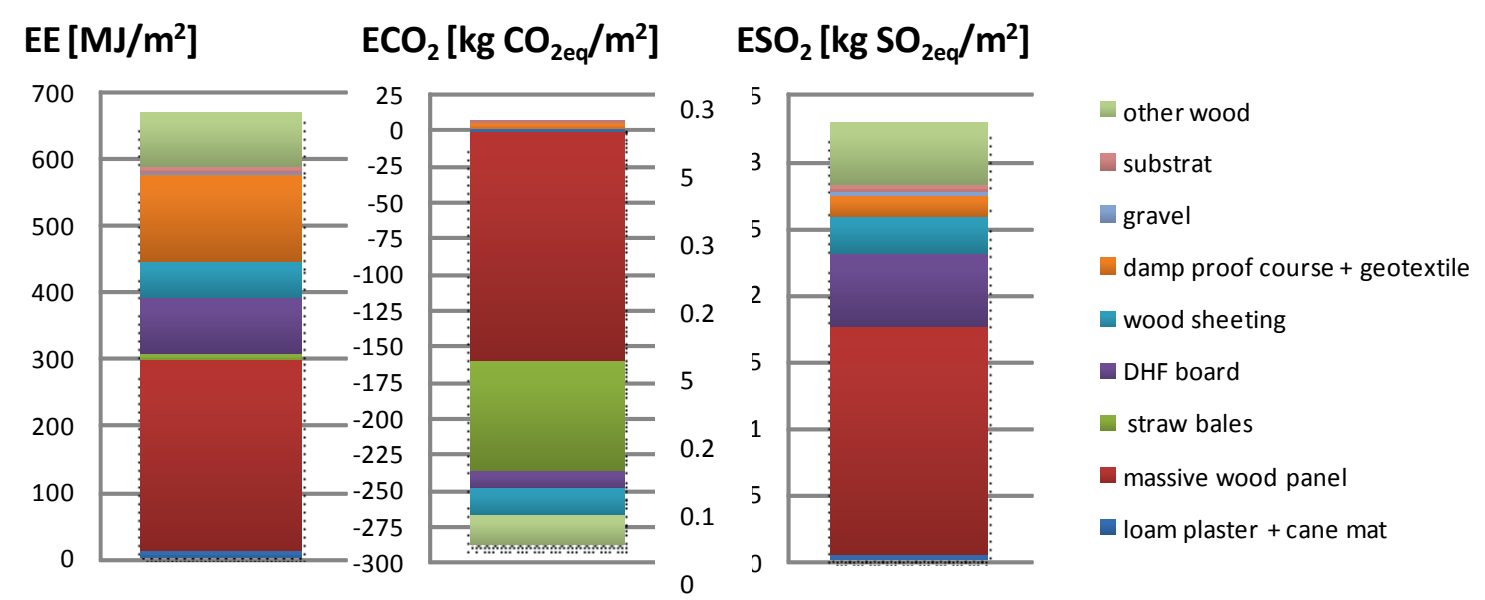

Figure 15. Roof structure: Impact of materials on environmental indicators.

Natural materials were used efficiently in vernacular houses. It is clear that when insulating habitats, the interrelated environmental impacts of the selection of insulation materials is not relevant compared to energy savings. Another study [44] analyzed the implications of various insulation materials on the primary energy and $\mathrm{CO}_{2}$ emissions for the material production of a residential building. The study compared the primary energy use and $\mathrm{CO}_{2}$ emissions from the material production of functionally equivalent reference buildings and optimum versions of the building. The results showed a reduction of about $6 \%-7 \%$ in primary energy use and $6 \%-8 \%$ in $\mathrm{CO}_{2}$ emissions when the rock wool insulation material in the reference buildings was changed to cellulose fiber in the optimum versions. According to a different study [45], the use of light clay bricks (85\% clay and 15\% straw) or silico-calcareous (90\% lime and 10\% sand) bricks clearly reduces impacts. Although in light clay bricks the primary energy demand is relatively high, it is important to note that $45 \%$ of this energy originates from biomass due to the straw content. In addition, light clay bricks have a practically neutral $\mathrm{CO}_{2}$ balance. It is important to underline that the impact of conventional insulation with a high level of industrial processing such as EPS is clearly higher than the impact of natural materials such as cork, wood fiber, and sheep's wool or recycled materials such as cellulose fiber.

Our study also shows that natural materials play a role in the reduction of environmental impacts. This is confirmed by the results from evaluation of external wall compositions designed from massive wood panels with straw, fleece, and fiberboard insulations (Table 7). A different study [46] indicated that fleece has low global warming potential. Alternative 18, the above-the-ground floor construction with fleece thermal insulation between I-profiles, was the best in terms of EE, $\mathrm{SO}_{2 \text { eq }}$ emissions, and OI3 Con environmental indicators (Figure 7) and mass per unit area (Figure 6). Another study [47] revealed that the variant with calcium silicate requires no vapor barrier or gypsum board and thus the overall design has a smaller $\mathrm{CO}_{2 \text { eq }}$ balance. In our study, Alternative 14 (roof structure composition) had maximum $\mathrm{CO}_{2 \text { eq }}$ values because of its aluminum vapor barrier and of aluminum sheet covering. A comprehensive LCIA of single-family houses in Portugal drew the conclusion that exterior walls were the most important construction component and contributed $35 \%$ of the EE and $43 \%$ of $\mathrm{CO}_{2 \mathrm{eq}}$ emissions associated with the construction phase. The comparative analysis of seven wall scenarios with different materials indicated that wood walls are the preferred solution and have the lowest impacts [45]. A different study [48] confirmed that timber is the most environmentally building material compared to others. The GWP of a timber house was determined to be $4.19 \mathrm{E}+02 \mathrm{~kg} \mathrm{CO}$, 
$3.85 \mathrm{E}+02 \mathrm{~kg} \mathrm{CO}$, and $3.68 \mathrm{E}+02 \mathrm{~kg} \mathrm{CO}$, respectively, for life spans of 20, 100, and 500 years. However, the low resistance of wood structures against load bearing (high humidity) in the Malaysian climate means that wood is not the first choice of house builders. Our study confirmed that based on MCDA, massive wood panels are a very effective material.

Currently, there exists no single insulation material or solution capable of fulfilling all the requirements with respect to the most crucial properties [49]. Among modern thermal insulation materials we have aerogels and vacuum insulation panels (VIP). Aerogel for building applications has a thermal conductivity of around $14-16 \mathrm{~mW} /(\mathrm{m} \cdot \mathrm{K})$ while VIP can reach down to $4 \mathrm{~mW} /(\mathrm{m} \cdot \mathrm{K})$. This can be compared to EPS and stagnant air with a thermal conductivity of 36 and $25 \mathrm{~mW} /(\mathrm{m} \cdot \mathrm{K})$ respectively. Aerogel and VIP have small pores in the range of 10-100 nm which means the gas conductivity is reduced at atmospheric pressure [50]. VIPs have thermal resistance values, at the centre of the panel, up to 10 times or more than those of conventional thermal insulation materials [51]. Energy efficient VIPs present thin but highly effective insulation solutions, to reduce thermal losses for both new and renovated buildings [52]. The environmental impact of transparent aerogel has been assessed in study [53]. Study [50] showed that VIP had a lower environmental impact than EPS which was $42 \%$ higher than VIP, while mineral wool was only half compared to VIP. The high figure for EPS was caused by the use of fossil based goods. The analysis showed that $90 \%$ of the energy used in VIP production derived from the core material while only $4 \%$ was used for the laminate production. As this study [50] state, there are a number of different novel thermal insulation materials and components which are used in the building industry, such as foamglass, polyurethane (PUR), polyisocyanurate (PIR) and polystyrene with added graphite. Review [49] presented a summary of the conventional and novel thermal insulation materials as gas-filled panels (GFP) and phase change materials (PCM). In the future is appears necessary to analyze more material compositions using modern thermal insulation materials in construction of nearly zero energy houses.

\section{Conclusions}

Wooden houses represent significant potential for eliminating the environmental burden in relation to the use of building materials and have a high predisposition for meet the criteria of green buildings. In recent years, wood has been increasingly used as a primary building material—not only because of environmental quality but also due to the possibility of a close link with nature and the creation of a healthy environment. This strategic renewable raw material can be seriously considered as the material of the third millennium.

Analysis and multi-criteria assessment of material compositions of structures confirmed that renewable natural materials produced by unpretending technologies are highly effective for the design of wooden houses that are simultaneously green buildings. Building structures consisting of natural materials exhibit low EE values due to the fact that solar energy is used in the production of primary materials. In addition, plant materials largely conserve carbon within their mass and thus contribute to the elimination of global warming. From environmental and thermo-physical viewpoints, structures consisting of massive wood, straw and clay are superior. These materials are locally available, and straw is an annually renewing agricultural waste product. At the end of the life of these building materials, they can be returned back to nature and used as a source of energy or, in some cases, in new 
construction. From a thermal-physical viewpoint, the diffusely open structures from cob brick, massive wood panels, and thermal insulation from straw bales can improve the thermal stability of wooden houses and meet requirements for the construction of nearly-zero-energy buildings. Construction of buildings from local natural materials creates the opportunity to use raw material and become energy self-sufficient.

The results of analysis of alternatives designed for the building envelopes of non-transparent structures and evaluation of their energy and environmental aspects through MCDA can be summarized as follows:

- The best external wall alternative is composed of wood cladding (0.022 m), DHF board (0.015 m), straw bales of $90 \mathrm{~kg} / \mathrm{m}^{3}(0.300 \mathrm{~m})$ between I-profiles, a massive wood panel $(0.200 \mathrm{~m})$, and loam plaster with cane $(0.020 \mathrm{~m})$.

- The best above-the-ground floor alternative is composed of cork board $(0.020 \mathrm{~m})$, adobe brick $(0.060 \mathrm{~m})$, vapor barrier, OSB 3 board $(0.036 \mathrm{~m})$, straw bales of $90 \mathrm{~kg} / \mathrm{m}^{3}(0.400 \mathrm{~m})$ between I-profiles, and a massive wood panel $(0.212 \mathrm{~m})$.

- The best on-the-ground floor alternative is composed of blocks of wood (0.012 m), cork (0.008 m), concrete slab $(0.200 \mathrm{~m})$, water proofing + geotextile, foam glass $(0.500 \mathrm{~m})$, separated geotextiles, and soil.

- The best roof structure alternative is composed of loam plaster with cane $(0.020 \mathrm{~m})$, a massive wood panel $(0.212 \mathrm{~m})$, straw bales of $90 \mathrm{~kg} / \mathrm{m}^{3}(0.400 \mathrm{~m})$ between I-profiles, DHF board $(0.015 \mathrm{~m})$, wood sheeting $(0.080 \mathrm{~m})$, water proofing + geotextiles, gravel $(0.030 \mathrm{~m})$, and substrate $(0.060 \mathrm{~m})$.

\section{Acknowledgments}

This study was supported by the Grant Agency of Slovak Republic via its support for project No. $1 / 0405 / 13$.

\section{Author Contributions}

Silvia Vilcekova contributed to the theoretical analysis of research problem, Monika Culakova proposed the material compositions and evaluation of alternatives, Eva Kridlova Burdova contributed to results evaluation from MCDA and Jana Katunska contributed to the proposal of compositions for building constructions.

\section{Conflicts of Interest}

The authors declare no conflict of interest. 


\section{Nomenclature}

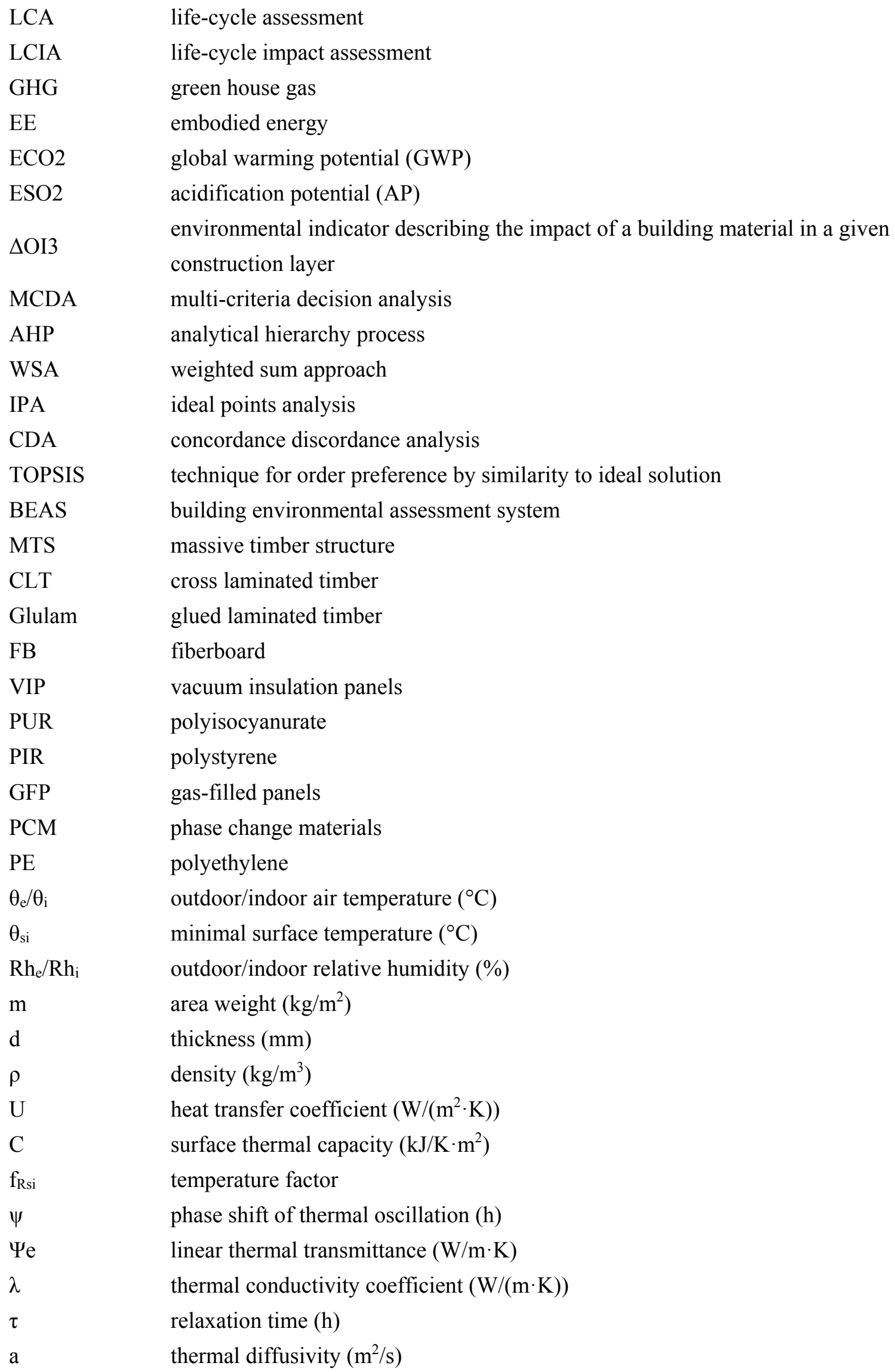




\section{References}

1. Neiva, S.; Mateus, R.; Bragança, L. Environmental LCA database for conventional Portuguese building Technologies. In Proceedings of the 1st International Conference on Building Sustainability Assessment, Porto, Portugal, 23-25 May 2012; pp. 613-620.

2. Cordero, R.R.; Roth, P.; da Silva, L. Economic growth or environmental protection? The false dilemma of the Latin-American countries. Environ. Sci. Policy 2005, 8, 392-398.

3. Akadiri, O.P. ICT Development of a multi-criteria approach for the selection of sustainable materials for building projects. Ph.D. Thesis, University of Wolverhampton, West Midlands, UK, February 2011; p. 437.

4. Energy, Transport and Environment Indicators. Eurostat. Pocketbooks. 2014. Available online: http://ec.europa.eu/eurostat/documents/3930297/6613266/KS-DK-14-001-EN-N.pdf/4ec0677e8fec-4dac-a058-5f2ebd0085e4 (accessed on 7 July 2015).

5. Dodoo, A.; Gustavsson, L.; Sathre, R. Building energy-efficiency standards in a life cycle primary energy perspective. Energy Build. 2011, 43, 1589-1597.

6. Balaras, C.A.; Gaglia, A.G.; Georgopoulou, E.; Mirasgedis, S.; Sarafidis, Y.; Lalas, D.P. European residential buildings and empirical assessment of the Hellenic building stock, energy consumption, emissions and potential energy savings. Build. Environ. 2007, 42, 1298-1314.

7. Poel, B.; Cruchtena, G.; Balaras, C.A. Energy performance assessment of existing dwellings. Energy Build. 2007, 39, 393-403.

8. Acquaye, A.A.; Duffy, A.P.; Basu, B. Stochastic hybrid embodied $\mathrm{CO}_{2}$-eq analysis: An application to the Irish apartment building sector. Energy Build. 2011, 43, 1295-1303.

9. Huberman, N.; Pearlmutter, D. A life-cycle energy analysis of building materials in the Negev desert. Energy Build. 2008, 40, 837-848.

10. Paleari, M.; Lavagna, M.; Campioli, A. Life cycle assessment of a zero energy residential building. In Proceedings of the 1st International Conference on Building Sustainability Assessment, Porto, Portugal, 23-25 May 2012; pp. 641-650.

11. Dimoudi, A.; Tompa, C. Energy and environmental indicators related to construction of office buildings. Resour. Conserv. Recycl. 2008, 53, 86-95.

12. Soares, N.; Costa, J.J.; Gaspar, A.R.; Santos, P. Review of passive PCM latent heat thermal energy storage systems towards buildings' energy efficiency. Energy Build. 2013, 59, 82-103.

13. Frenette, C.D.; Bulle, C.; Beauregard, R.; Salenikovich, A.; Derome, D. Using life cycle assessment to derive an environmental index for light-frame wood wall assemblies. Build. Environ. 2010, 45, 2111-2122.

14. Monahan, J.; Powell, J.C. An embodied carbon and energy analysis of modern methods of construction in housing: A case study using a lifecycle assessment framework. Energy Build. 2011, 43, 179-188.

15. Sartori, I.; Hestnes, A.G. Energy use in the life cycle of conventional and low energy buildings: A review article. Energy Build. 2007, 39, 249-257.

16. Scheuer, C.; Keoleian, G.A.; Reppe, P. Life cycle energy and environmental performance of a new university building. Energy Build. 2003, 35, 1049-1064. 
17. Petersen, A.; Solberg, B. Greenhouse gas emissions, life-cycle inventory and cost-efficiency of using laminated wood instead of steel construction. Environ. Sci. Policy 2002, 5, 169-182.

18. Thormark, C. The effect of material choice on the total energy need and recycling potential of a building. Build. Environ. 2006, 41, 1019-1026.

19. Stephan, A.; Crawford, R.H.; Myttenaere, K. Towards a comprehensive life cycle energy analysis framework for residential buildings. Energy Build. 2012, 55, 592-600.

20. Coelho, A.C.; Branco, J.M.; Gervásio, H. Life-cycle assessment of a single-family timber house. In Proceedings of the 1st International Conference on Building Sustainability Assessment, Porto, Portugal, 23-25 May 2012; pp. 533-542.

21. Borjesson, P.; Gustavsson, L. Greenhouse gas balances in building construction: Wood versus concrete from life-cycle and forest land-use perspectives. Energy Policy 2000, 28, 575-588.

22. Liu, M.; Li, B.; Yao, R. A generic model of exergy assessment for the environmental impact of building lifecycle. Energy Build. 2010, 42, 1482-1490.

23. Monteiro, H.; Freire, F. Environmental life-cycle impacts of a single-family house in Portugal. Gazi Univ. J. Sci. 2011, 24, 527-534.

24. Verbeeck, G.; Hens, H. Life cycle inventory of buildings: A contribution analysis. Build. Environ. 2010, 45, 964-967.

25. Cuéllar-Franca, R.M.; Azapagic, A. Environmental impacts of the UK residential sector: Life cycle assessment of houses. Build. Environ. 2012, 54, 86-99.

26. Blengini, G.A.; Carlo, T.D. The changing role of life cycle phases, subsystems and materials in the LCA of low energy buildings. Energy Build. 2010, 42, 869-880.

27. Zhang, X.; Wang, F. Life-cycle assessment and control measures for carbon emissions of typical buildings in China. Build. Environ. 2015, 86, 89-97.

28. Airaksinen, M.; Matilainen, P. A carboon footprint of an office building. Energies 2011, 4, 1197-1210.

29. Heinonen, J.; Säynäjoki, A.; Kuronen, M.; Junnila, S. Are the greenhouse gas implications of new residential developments understood wrongly? Energies 2012, 5, 2874-2893.

30. Cole, R.J. Energy and greenhouse gas emissions associated with the construction of alternative structural systems. Build. Environ. 1999, 34, 335-348.

31. Suzuki, M.; Oka, T.; Okada, K. The estimation of energy consumption and $\mathrm{CO}_{2}$ emission due to housing construction in Japan. Energy Build. 1995, 22, 165-169.

32. Takano, A.; Pal, S.K.; Kuittinen, M.; Alanne, K. Life cycle energy balance of residential buildings: A case study on hypothetical building models in Finland. Energy Build. 2015, 105, 154-164.

33. Estokova, A.; Porhincak, M. Environmental analysis of two building material alternatives in structures with the aim of sustainable construction. Clean. Technol. Environ. Policy 2015, 17, 75-83.

34. Hui, S.C.M. Low energy building design in high density urban cities. Renew. Energy 2001, 24, 627-640.

35. Sedláková, A.; Vilčeková, S.; Burdová, E.K. Analysis of material solutions for design of construction details of foundation, wall and floor for energy and environmental impacts. Clean. Technol. Environ. Policy 2015, 17, 1323-1332. 
36. Čuláková, M.; Vilčeková, S.; Hejhálek, J. Assessment of performance of building structures in compliance with sustainable concept. In Advances in Civil, Structural and Environmental Engineering (ACSEE): Proceedings of the International Conference; Institute of Research Engineers and Doctors: Zurich, Switzerland; Newark, DE, USA, 2013; pp. 103-106.

37. BRE Environmental Profiles 2013. Product Category Rules for Type III environmental product declaration of construction products to EN 15804:2012. Final version. Available online: https://www.bre.co.uk/filelibrary/BRE_EP_15804_PCR_final_version_II_for_public_consultation.p df (accessed on 30 September 2015).

38. De Benedetto, D.; Klemeš, J. The environmental performance strategy map: An integrated LCA approach to support the strategic decision-making process. J. Clean. Prod. 2009, 17, 900-906.

39. Waltjen, T. Passivhaus-Bauteilkatalog, Ökologisch bewertete Konstruktionen; Springer: Wien, Austria, 2009.

40. OI3-indicator: Leitfaden zur Berechnung von Ökokennzahlen für Gebäude. (2011). Available online: http://www.ibo.at/documents/OI3_Berechnungsleitfaden_V3.pdf (accessed on 7 July 2015).

41. Wang, J.-J.; Jing, Y.-Y.; Zhang, C.-F.; Zhao, J.-H. Review on multi-criteria decision analysis aid in sustainable energy decision-making. Renew. Sustain. Energy Rev. 2009, 13, 2263-2278.

42. Korviny, P. Theoretical Basis of Multi-Criteria Decision. Ph.D. Thesis, Technical University of Ostrava, Ostrava, Czech Republic, 2009.

43. Almusaed, A.; Almssad, A. Building materials in eco-energy houses from Iraq and Iran. Case studies in construction. Materials 2015, 2, 42-54

44. Tettey, U.Y.A.; Dodoo, A.; Gustavsson, L. Effects of different insulation materials on primary energy and $\mathrm{CO}_{2}$ emission of a multi-storey residential building. Energy Build. 2014, 82, 369-377.

45. Zabalza, B.I.; Capilla, A.V.; Usón, A.A. Life cycle assessment of building materials: Comparative analysis of energy and environmental impacts and evaluation of the eco-efficiency improvement potential. Energy Build. 2011, 46, 1133-1140.

46. Korjenic, A.; Klarić, S.; Hadžić, A.; Korjenic, S. Sheep wool as a construction material for energy efficiency improvement. Energies 2015, 8, 5765-5781.

47. Monteiro, H.; Freire, F. Life-cycle assessment of a house with alternative exterior walls: Comparison of three impact assessment method. Energy Build. 2012, 47, 572-583.

48. Marsono, A.K.B.; Balasbaneh, A.T. Combinations of building construction material for residential building for the global warming mitigation for Malaysia. Construct. Build. Mater. 2015, 85, $100-108$.

49. Jelle, B.P. Traditional, state-of-the-art and future thermal building insulation materials and solutions - Properties, requirements and possibilities. Energy Build. 2011, 43, 2549-2563.

50. Berge, A.; Johansson, P. Literature Review of High Performance Thermal Insulation. Report in Building Physics; Chalmers University of Technology: Gothenburg, Sweden, 2012.

51. Mukhopadhyay, P.; MacLean, D.; Korn, J.; van Reenen, D.; Molleti, S. Building application and thermal performance of vacuum insulation panels (VIPs) in Canadian subarctic climate. Energy Build. 2014, 85, 672-680. 
52. Boafo, F.E.; Chen, Z.; Li, C.; Li, B.; Xu, T. Structure of vacuum insulation panel in building system. Energy Build. 2014, 85, 644-653.

53. Dowson, M.; Grogan, M.; Birks, T.; Harrison, D.; Craig, S. Streamlined life cycle assessment of transparent silica aerogel made by supercritical drying. Appl. Energy 2012, 97, 396-404.

(C) 2015 by the authors; licensee MDPI, Basel, Switzerland. This article is an open access article distributed under the terms and conditions of the Creative Commons Attribution license (http://creativecommons.org/licenses/by/4.0/). 